Article

\title{
Paleoenvironmental Conditions during the Paleocene-Eocene Transition Imprinted within the Glauconitic Giral Member of the Barmer Basin, India
}

\author{
Tathagata Roy Choudhury ${ }^{1}(\mathbb{D})$, Santanu Banerjee ${ }^{1, *}(\mathbb{C})$, Sonal Khanolkar ${ }^{2}(\mathbb{C})$ and Sher Singh Meena ${ }^{3}(\mathbb{C})$ \\ 1 Department of Earth Sciences, Indian Institute of Technology, Mumbai 400076, India; tathagata_rc@iitb.ac.in \\ 2 Department of Climate Geochemistry, Max Planck Institute for Chemistry, 55128 Mainz, Germany; \\ sonal.k.12@gmail.com \\ 3 Solid State Physics Division, Bhabha Atomic Research Centre, Mumbai 400085, India; ssingh@barc.gov.in \\ * Correspondence: santanu@iitb.ac.in
}

check for updates

Citation: Roy Choudhury, T.;

Banerjee, S.; Khanolkar, S.; Meena, S.S. Paleoenvironmental Conditions during the Paleocene-Eocene Transition Imprinted within the Glauconitic Giral Member of the Barmer Basin, India. Minerals 2022, 12, 56. https://doi.org/10.3390/ $\min 12010056$

Academic Editor: Hermann Kudrass

Received: 8 November 2021

Accepted: 30 December 2021

Published: 31 December 2021

Publisher's Note: MDPI stays neutral with regard to jurisdictional claims in published maps and institutional affiliations.

Copyright: (c) 2021 by the authors. Licensee MDPI, Basel, Switzerland. This article is an open access article distributed under the terms and conditions of the Creative Commons Attribution (CC BY) license (https:/ / creativecommons.org/licenses/by/ $4.0 /)$.

\begin{abstract}
The roughly $6 \mathrm{~m}$ thick limestone-green shale alternation within the lignite-bearing Giral Member of the Barmer Basin corresponds to a marine flooding event immediately after the PaleoceneEocene transition. A detailed characterization of the glauconite using Electron Probe Micro Analyzer (EPMA), X-Ray Diffraction (XRD), Mössbauer and Field Emission Gun-Scanning Electron Microscope (FEG-SEM) reveals its origin in the backdrop of prevailing warm climatic conditions. The glauconite pellets vary from fine silt-sized to coarse sand-sized pellets, often reaching $\sim 60 \%$ of the rock by volume. Mineralogical investigation reveals a 'nascent' to 'slightly evolved' character of the marginal marine-originated glauconite showing considerable interstratification. The chemical composition of the glauconite is unusual with a high $\mathrm{Al}_{2} \mathrm{O}_{3}(>10 \mathrm{wt} \%)$ and moderately high $\mathrm{Fe}_{2} \mathrm{O}_{3}$ (total) contents $(>15 \mathrm{wt} \%)$. While the $\mathrm{K}_{2} \mathrm{O}$ content of these glauconites is low, the interlayer sites are atypically rich in $\mathrm{Na}_{2} \mathrm{O}$, frequently occupying $\sim 33 \%$ of the total interlayer sites. The Mössbauer spectrum indicates $10 \%$ of the total iron is in ferrous form. High tetrahedral $\mathrm{Al}^{3+}$ of these glauconites suggests a high-alumina substrate that transformed to glauconite by octahedral Al-for-Fe substitution followed by the addition of $\mathrm{K}$ into the interlayer structure. The unusually high $\mathrm{Na}_{2} \mathrm{O}$ suggests the possibility of a soda-rich pore water formed by the dissolution of alkaline volcanic minerals. The Giral glauconite formation could have been a part of the major contributors in the Fe-sequestration cycle in the Early Eocene shelves. Warm climate during the Early Eocene time favored the glauconitization because of the enhanced supply of $\mathrm{Fe}, \mathrm{Al}$, and $\mathrm{Si}$ and proliferation of an oxygen-depleted depositional environment.
\end{abstract}

Keywords: glauconite; lignite; authigenic mineral formation; PETM; Barmer Basin; shallow marine; iron sequestration

\section{Introduction}

Glauconite occurs as rounded to sub-rounded, light-green to dark-green pellets that form on the sediment-seawater interface [1-16]. The evolution of early formed glauconite leads to widely varying compositional range from an interlayer-deficient, K-poor glauconitic smectite to K-rich glauconitic mica [1], within which the interlayer Na content rarely exceeds $1 \mathrm{wt} \%$. The term 'glauconite' is used for the minerals with end-member composition having $\mathrm{K}_{2} \mathrm{O}>8 \mathrm{wt} \%$ and $<5 \%$ smectite interstratification while the term glaucony incorporates the wide compositional range from $2-8 \mathrm{wt} \% \mathrm{~K}_{2} \mathrm{O}$ and $>5 \%$ smectite interstratification [1]. Because of the fact that these terminologies can be used interchangeably, in this paper we have used the term 'glauconite' for glauconitic mineral composition with considerable smectite interstratification. Understanding the authigenesis of glauconite is critical in modelling the fluxes in the global iron cycle [17]. Whereas the supply of iron is primarily linked to the continental input, iron sequestration occurs primarily in marine conditions. In shallow marine, sub-oxic depositional environments, glauconite authigenesis 
plays an important role in iron sequestration [17]. Authigenic glauconite occurs profusely within the Paleogene sedimentary deposits across the globe [18]. Contrary to modern glauconites which preferably form on the outer shelf and continental slope, the Paleogene glauconites are associated with shallow marine environments [7,19]. High-resolution biostratigraphic data of the Paleogene deposits establishes a close association between glauconite occurrence and hyperthermal events [18-21]. Roy Choudhury et al. [19] reported unique glauconite with high $\mathrm{Al}_{2} \mathrm{O}_{3}$ and moderately high $\mathrm{Fe}_{2} \mathrm{O}_{3}$ (total) contents within the middle shelf deposits of the Jaisalmer Basin, India, and documented its relationship with the hyperthermal event Paleocene Eocene Thermal Maximum (PETM). A detailed study of the glauconites of similar ages is thus necessary to refine our understanding regarding glauconitization during warm climatic intervals.

Paleogene deposits of India have received particular importance for the economically viable lignite hydrocarbon deposits [22]. Recent studies reveal glauconite deposits within all the Paleogene successions of India. Glauconite occurs within the marginal marine to shallow shelf deposits of Kutch, Cambay, Jaisalmer and Assam-Arakan basins [5,6,19,23-25]. Recent studies establish several hyperthermal events within the Paleogene deposits, enabling the study of glauconite authigenesis in the backdrop of warm climatic conditions [26-28]. The Barmer Basin in western India represents a restricted marginal marine basin, which is separated from the Jaisalmer Basin by the Devikot High [29-31]. Paleogene deposits of the Barmer Basin include coarse-grained sandy sediments succeeded upward by the alternation of lignite and fine-grained shale, containing the glauconite. This study focuses on the Early Eocene glauconite and aims to (a) highlight the unique geochemical and mineralogical characteristics of glauconite formed during the early Paleogene time, and (b) to consider the factors influencing the composition of glauconite, in the backdrop of warm climatic conditions.

\section{Geological Background}

The Meso-Cenozoic succession of the Barmer Basin unconformably overlies the Proterozoic Malani Igneous Suite, which represents the basement at the northern part of the basin. The studied section belongs to the Paleogene lignite-bearing deposit of the Barmer Basin around the Giral area (Figure 1A). The Barmer Basin is an NW-SE-trending failed rift showing three major stages of basin fills, viz., Jurassic to Cretaceous pre-rift, Early Paleocene to early Eocene syn-rift, and younger post-rift deposits [31]. The syn-rift succession initiates with the deposition of the Fatehgarh Sandstone, which is dominated by quartzose sandstone and conglomerate with thin phosphatic and ferruginous sandstone intercalations [30,32]. The Fatehgarh Sandstone is overlain by the Barmer Hill Formation, represented by a thick lacustrine shale with intermittent turbidite deposits [31]. The syn-rift Barmer Hill Formation is unconformably overlain by the Dharvi Dungar and Thumbli Formation, comprised of alternations of shale and lignite [31]. Both Dharvi Dungar and Thumbli Formations consist of cyclic alternation of claystone, siltstone with occasional sandstones, and intermittent limestones. The lignite deposits within the Giral lignite Mine belong to the Giral Member of the Dharvi Dungar Formation [33]. Seismic sections reveal the unconformable contact between the Barmer Hill Formation and the Dharvi Dungar Formation [31]. A few workers considered the lignite-bearing succession as the Akli Formation [32,34-37]. In this study, we have adopted the stratigraphic scheme of Dolson et al. [31] and considered the lignite-bearing succession of the Giral Mine as the Giral Member of the Dharvi Dungar Formation. 


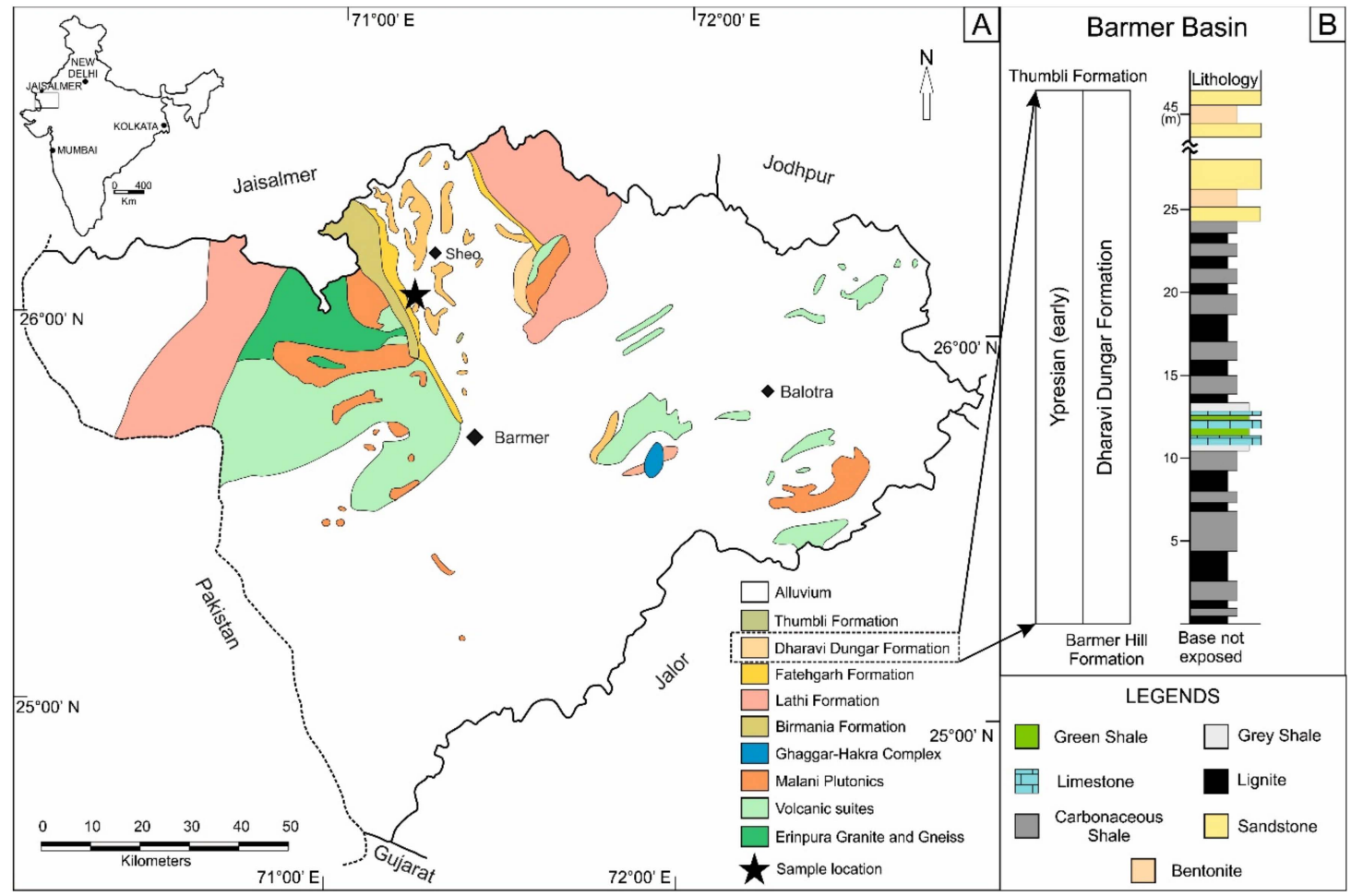

Figure 1. (A) Detailed geological map of the Barmer Basin (area marked by rectangle on the Indian map) (modified after Rana et al. [34]) with necessary stratigraphic elaborations. The asterisk marks the location of the Giral Lignite Mine; (B) lithocolumn showing alternating lignite-shale succession interrupted by a limestone-green shale alternation within the Giral Member.

The age of the lignite-bearing succession of the Giral Member is assessed by floral and faunal assemblages including palynomorphs, vertebrate fauna, and benthic foraminifera. The basal part of the Giral Member has yielded vertebrate fossils such as fish, crocodiles, and snakes which were assigned broadly to be of late Paleocene-early Eocene age [34]. The rich palynofloral assemblage of the Giral Member includes a high abundance of dinoflagellate cysts, pteridophytic spores, and angiosperm pollens [29,38]. The presence of dinocyst assemblage defined by Apectodinium homomorphum, Adnatosphaeridium sp., Glaphyrocysta exuberans, Kenleyia sp., and Thalassiphora pelagica within the shales suggests an early Eocene (Ypresian) age for the lignite deposits of the Giral Member [33,39]. Although the dinocyst assemblage is not a precise age indicator, the same in the adjacent Cambay Basin and other parts of the world marks the earliest part of the Eocene, immediately after the Paleocene-Eocene Thermal Maximum (PETM).

\section{Materials and Methods}

Barmer Basin deposits are well-studied using subsurface data as the outcrops are covered with desert sands. The Giral Member, exposing the Cenozoic succession, hosts the lignite deposit. The present findings are based on investigations in the Giral Lignite Mine $\left(27^{\circ} 7^{\prime} 34.54^{\prime \prime} \mathrm{N}, 70^{\circ} 29^{\prime} 37.06^{\prime \prime} \mathrm{E}\right)$, located $\sim 45 \mathrm{~km}$ northwest of Barmer. Encased with lignite seams, greenish grey shale alternates with argillaceous limestone at the mid-level of the quarry section (Figure 1). Four (4) samples were collected from the greenish grey shale from the same horizon at different parts of the mine and named as Gl/2a, Gl/2b, $\mathrm{Gl} / 2 \mathrm{c}$, and $\mathrm{Gl} / 2 \mathrm{~d}$. The greenish grey shale samples were epoxy-cured for thin section preparation. The hardened samples were cut and polished on Buehler@ (Buehlar Ltd., Lake Bluff, Illinois, USA) silicon carbide grinding and polishi ng paper and mounted on glass slides. The mounted chip was finally ground and polished to a thickness close to $0.03 \mathrm{~mm}$. Thin sections were examined using a Leica DM 4500P polarizing microscope at the Indian Institute of Technology (IIT) Bombay. X-ray diffraction analysis was carried 
out separately on the clay fraction ( $<2 \mu \mathrm{m}$ grain size) and on separated glauconite grains. The clay fraction was obtained by gravitative settling of the bulk rock powder (finer than 240 sieve in the American Society for Testing and Materials (A.S.T.M.) in a $100 \mathrm{~mL}$ glass cylinder followed by centrifugation of the suspended water column for 15-20 min at $3000 \mathrm{rpm}$. The clay fraction obtained after centrifugation was air-dried and analyzed as randomly-oriented pressed powder pellets. Glauconite pellets were separated from the whole rock by heating the lightly crushed samples with $\mathrm{Na}_{2} \mathrm{CO}_{3}$ solution, followed by sieving (500 ASTM). Glauconite pellets were handpicked using a Zeiss Stemi 2000 stereozoom microscope for XRD analysis. The pellets were smear-mounted on a glass slide for oriented sample preparation. The clay fraction and the oriented, smear-mounted glauconite were scanned from $4^{\circ}$ to $70^{\circ}$ with a step size of $0.026^{\circ} 2 \theta$, scan speed of $96 \mathrm{~s} / \mathrm{step}$, using nickel filter copper radiation in an Empyrean X-ray diffractometer with Pixel 3D detector at IIT Bombay. The oriented glauconite pellets were further scanned each time after air-drying, treated with ethylene glycol $\left(100{ }^{\circ} \mathrm{C}\right.$ for $\left.1 \mathrm{~h}\right)$, and heated under $400-500{ }^{\circ} \mathrm{C}$ temperature using the same instrumental settings. Major oxide analysis of the glauconite grain was carried out using a Cameca SX 5 Electron Probe Micro Analyzer at the Department of Earth Sciences, IIT Bombay, with accelerating voltage $15 \mathrm{kV}$, specimen current $40 \mathrm{nA}$, and a beam diameter $1 \mu \mathrm{m}$ (peak: 10-20 s and background counting: 5-10 s). Minerals and synthetic phases were used as standards. Duplicate analysis of individual points shows an analytical error of less than 1\%. Mössbauer spectra of glauconite samples were measured at the Solid State Physics Division, Bhabha Atomic Research Centre (BARC), Mumbai. A $\gamma$-ray source of ${ }^{57} \mathrm{Co}$ in Rh matrix at room temperature was used. An $\alpha$-Fe absorber was used at room temperature to calibrate the Doppler velocity (V) and the standard for the isomer shift (IS).

\section{Results and Interpretation}

\subsection{Sedimentological Background}

The Giral Lignite Mine exposes a $\sim 45$ thick section of the Giral Member containing 11 lignite seams. The boundary between the Barmer Hill and Dharvi Dungar formations is not exposed within the mine section. The Giral Member comprises four lithofacies, viz., lignite, carbonaceous shale, green shale, and argillaceous limestone. The lignite-shalelimestone bearing succession of the Giral Member succeeds upward by 20-25 m thick alternation of bentonite and sandstone. The thick cover of desert sands prevents tracing of the succession outside the mine area.

Lignite is the major constituent of the Giral Member of the Dharvi Dungar Formation. It appears as dark-brown to black seams varying in thickness from $30 \mathrm{~cm}$ to $1.5 \mathrm{~m}$ (Figure 2A-C). Lignites contain leaf imprints and minor amber/resin. The lignite seams alternate with carbonaceous shale varying in thickness from several $\mathrm{cm}$ to up to $2 \mathrm{~m}$ (Figure 2A,B,D). The carbonaceous shale imparts a dark grey color due to the high content of organic carbon. Leaf imprints are also common within these facies. Occasionally, the lignite seams pinch out within carbonaceous shale (Figure 2B). The thickness of the lignite seams varies with depth, being thicker at the bottom and top parts than the rest of the succession. The middle part of the succession is dominated by alternations between limestone and shale. This limestone-shale association ( $\sim 6 \mathrm{~m}$ thick) consists of nodular to bedded grey shale, impure limestone, and green shale and is laterally extensive along the Mine section. The thickness of the limestone bed varies between 10 to $15 \mathrm{~cm}$ while the green and grey shale varies from 30 to $50 \mathrm{~cm}$. The limestone exhibits rounded to ellipsoidal diagenetic nodules at places (Figure 2E). The grey shale, underlying the argillaceous limestone, shows wavy siltstone intercalation (Figure 2C). Grey shale contains leaf imprints and pyrite nodules in many places. The green shale contains glauconite pellets and alternates with thin siltstone beds, which vary in thickness from a few $\mathrm{mm}$ to $0.5 \mathrm{~cm}$. Glauconite pellets often comprise $50-60 \%$ of rock by volume, imparting the green color to the rock. As the content of glauconite decreases, the green shale changes to grey shale. Marine fossils, including dinoflagellate cysts, gastropods, and brachiopods along marine vertebrate fauna, are reported from the limestone-shale interval [33,34]. 

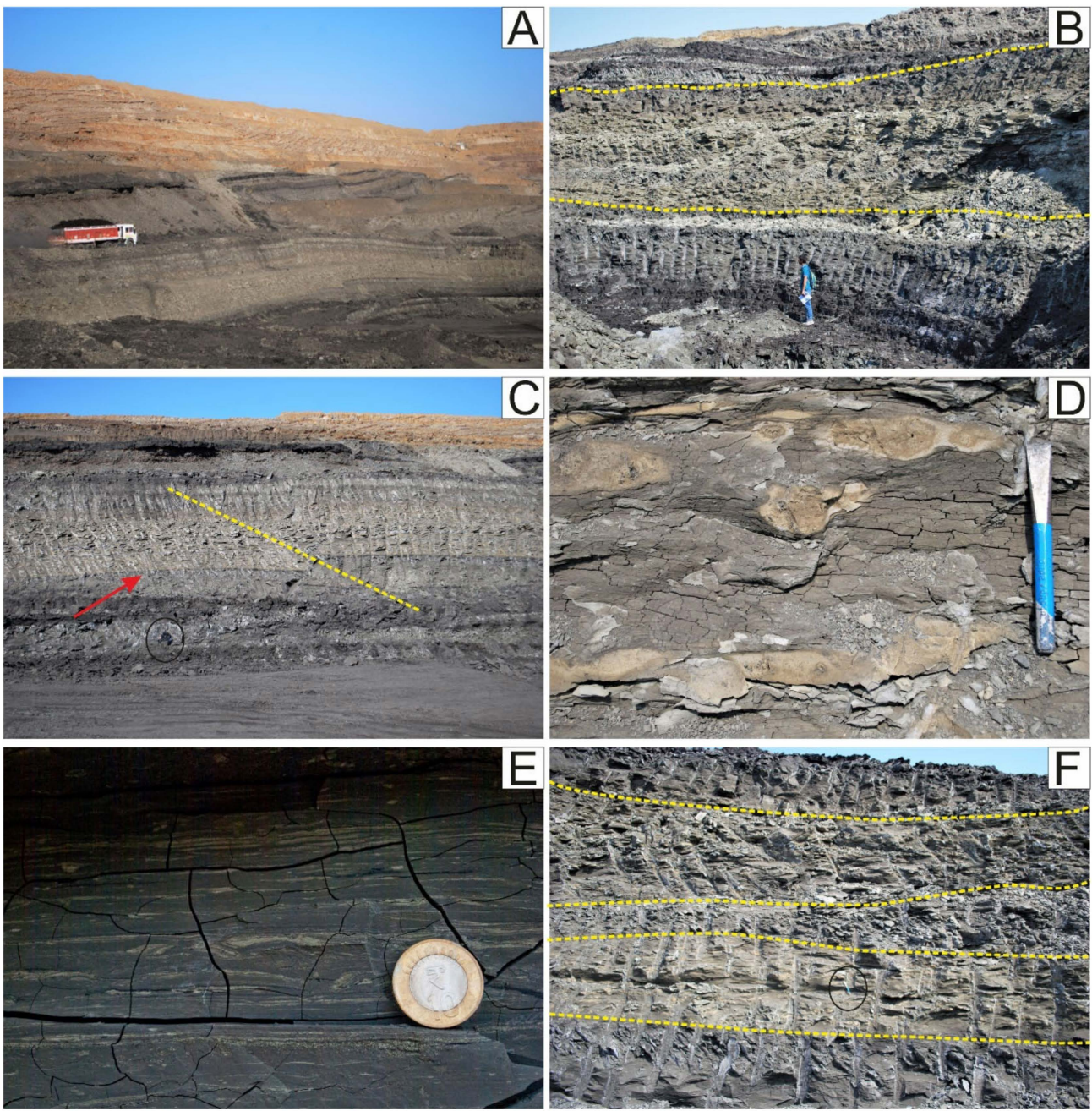

Figure 2. (A) Field photograph showing exposures of the Giral Member at the Giral Lignite Mine. (B) Vertical section exposing green shale-argillaceous limestone alteration (bounded by dotted lines). (C) The vertical section the Giral Member exposing thin lignite seams and the green shale-argillaceous limestone alternation (marked by red arrow); small-scale faults (marked with dotted line) often cause lateral variation of lignite thickness (black circle refers to scale representing $45 \mathrm{~cm}$ in height). (D) Vertical section showing diagenetic nodules within argillaceous limestone. (E) Vertical section exposing siltstone intercalations within the grey shale immediately below the limestone-green shale alternation; note the paucity of coarse-grained sediments. (F) Vertical section exposing the thickest $(\sim 90 \mathrm{~cm})$ limestone-green shale alternation on the western flack of the mine (black circle refers to scale representing $25 \mathrm{~cm}$ height).

The carbonaceous shale-lignite association of the Giral Member is dominated by the palynofloral assemblage representing tropical mangrove vegetation [33]. The abundance of Nypa pollen indicates its vicinity to paleoshoreline [33]. The alternation between argillaceous limestone, grey shale and green shale, with abundant dinoflagellate cyst and marine palynomorphs at the middle of the section, represents marine flooding [31-34]. The absence of wave-generated structures and the paucity of coarse-grained sediments within the succession corroborates the low-energy depositional conditions. An overall restricted marine depositional setting is indicated for the glauconitic interval of the Giral Member.

\subsection{Petrography of Glauconite}

The green shale facies exhibits a variable concentration of glauconite pellets, ranging from $\sim 20 \%$ to $\sim 60 \%$ within a fine-grained clayey matrix. Besides glauconite, detrital quartz and feldspar grains are also found within the clayey matrix of the green shale (Figure 3A). 
The size and shape of glauconite pellets are variable. The long dimension of the glauconite pellet ranges from $\sim 100 \mu \mathrm{m}$ to $\sim 700 \mu \mathrm{m}$ (av. $420 \mu \mathrm{m}$ ), with a few pellets exceeding $1000 \mu \mathrm{m}$. Pellets appear light green to dark green under plane polarized light and show mottled extinction under crossed polars (Figure 3A-C). Most of the pellets are rounded to subrounded, containing radial cracks within them (Figure 3B). A few pellets are rectangular, exhibiting sharp edges (Figure 3C). Radial cracks often break the pellets into small parts which are fitted together. A few elongated grains, showing variable interference color, appear as altered mica (Figure 3D). A few pellets resemble infillings within bioclasts, although no calcitic test has been observed within the green shale. Fine silt- to sand-sized quartz grains often show undulose extinction (Figure 3F). Many of the quartz grains have straight boundaries (Figure 3C,F). Lithoclasts, mostly containing feldspar with perthitic texture and small angular quartz grains occur in the green shale (Figure 3E). Incipient glauconite may form occasionally along the cleavage planes of feldspars (Figure 3F). Petrographic characteristics suggest authigenic glauconite formation. Preservation of delicate radial cracks within glauconite pellets confirms in situ formation $[1,5,6,19,40,41]$. The poorly sorted, euhedral quartz, and lithic fragments (Figure 3C-F) suggest a volcanic source for the detrital sediment [42].
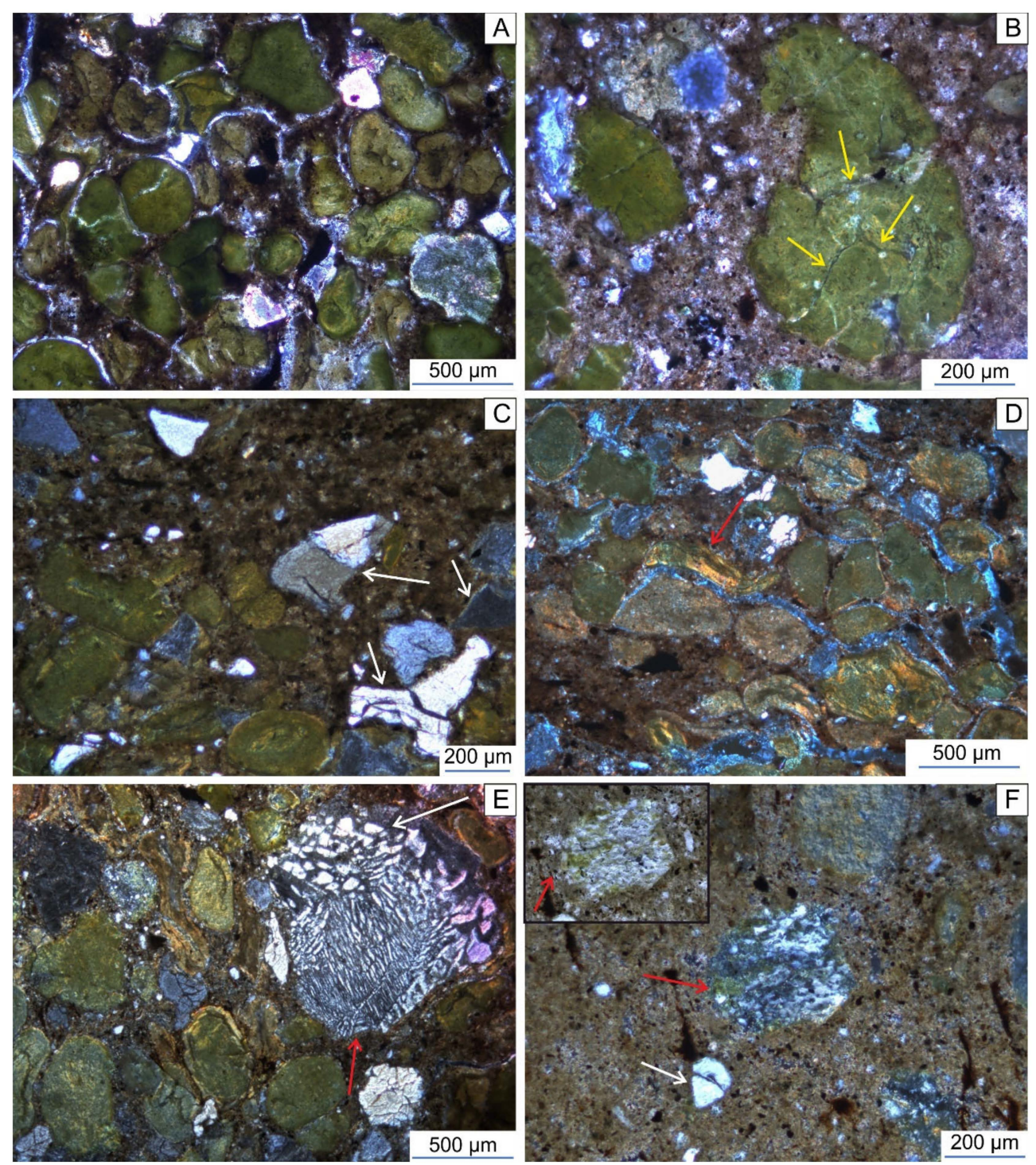

Figure 3. Photomicrographs of the green shale facies under cross-polarized light showing (A) high abundance of glauconite pellets of variable size and morphology; (B) glauconite pellet with abundant 
radical cracks (yellow arrows); (C) glauconite pellets associated with sub-angular to euhedral quartz grains with straight boundaries (white arrows); (D) showing elongated glauconite pellets with variegated interference color (red arrow); (E) volcanic lithoclast comprised of large and small angular to euhedral quartz grains (white arrow) and large feldspar grain with perthite texture (red arrow); and (F) incipient glauconite along the cleavage planes of feldspar (red arrow) and the euhedral quartz grain (white arrow) (inset figure shows incipient glauconite formation along feldspar cleavages (red arrow) in plane-polarized light).

\subsection{Major Oxide Concentrations of Glauconites}

The major element concentration of glauconite was obtained at 125 points in 4 thin sections (Table 1). The $\mathrm{K}_{2} \mathrm{O}$ content of the glauconite is low, varying between 3.09-5.52 wt\% (av. $4.14 \mathrm{wt} \%$ ). The $\mathrm{Na}_{2} \mathrm{O}$ content of the glauconite is high, varying from $0.22-1.90 \mathrm{wt} \%$ (av. $1.33 \mathrm{wt} \%$ ). The $\mathrm{Fe}_{2} \mathrm{O}_{3}$ (total) content of the pellets show a wide range, varying from 16.29 to $24.22 \mathrm{wt} \%$ (av. $19.88 \mathrm{wt} \%$ ). The $\mathrm{Al}_{2} \mathrm{O}_{3}$ content of most of the pellets varies between 9.87 and $16.77 \mathrm{wt} \%$ (av. $13.03 \mathrm{wt} \%$ ). The $\mathrm{SiO}_{2}$ content of the pellets varies from 48.48 to $57.20 \mathrm{wt} \%$ (av. $53.75 \mathrm{wt} \%$ ). The $\mathrm{MgO}$ content of the glauconites varies from 2.06 to $4.47 \mathrm{wt} \%$ (av. $2.66 w t \%$ ). Concentrations ( $w t \%$ ) of $\mathrm{CaO}, \mathrm{MnO}, \mathrm{TiO}_{2}$, and $\mathrm{P}_{2} \mathrm{O}_{5}$ are always less than $1 \mathrm{wt} \%$.

Table 1. Major oxide concentration of glauconites of the Giral Member, Dharvi Dungar Formation. (The mean and range of major oxides are provided for every sample).

\begin{tabular}{|c|c|c|c|c|c|c|c|c|c|c|c|c|}
\hline $\begin{array}{l}\text { DataSet } \\
\text { /Point }\end{array}$ & $\mathrm{Na}_{2} \mathrm{O}$ & $\mathrm{K}_{2} \mathrm{O}$ & $\mathrm{MgO}$ & $\mathrm{CaO}$ & $\mathrm{MnO}$ & $\mathrm{Al}_{2} \mathrm{O}_{3}$ & $\mathrm{FeO}$ & $\mathrm{Fe}_{2} \mathrm{O}_{3}$ & $\mathrm{SiO}_{2}$ & $\mathrm{TiO}_{2}$ & Total & $\begin{array}{c}\text { Sample } \\
\text { No. }\end{array}$ \\
\hline $1 / 1$ & 1.65 & 4.43 & 2.61 & 0.26 & 0.15 & 11.61 & 1.99 & 19.92 & 53.31 & 0.21 & 96.21 & \\
\hline $3 / 1$ & 1.55 & 4.46 & 2.62 & 0.25 & 0.06 & 11.32 & 1.99 & 19.93 & 53.80 & 0.38 & 96.39 & \\
\hline $4 / 1$ & 1.51 & 4.25 & 2.53 & 0.22 & 0.13 & 11.70 & 1.95 & 19.47 & 55.48 & 0.25 & 97.50 & \\
\hline $5 / 1$ & 1.72 & 3.81 & 2.70 & 0.28 & 0.08 & 14.55 & 1.65 & 16.48 & 55.20 & 0.39 & 96.91 & \\
\hline $6 / 1$ & 1.71 & 3.18 & 2.70 & 0.34 & 0.05 & 14.76 & 1.53 & 15.31 & 56.73 & 0.33 & 96.77 & \\
\hline $7 / 1$ & 1.50 & 3.86 & 2.62 & 0.24 & 0.11 & 14.77 & 1.57 & 15.68 & 55.88 & 0.44 & 96.69 & \\
\hline $8 / 1$ & 1.58 & 4.29 & 2.67 & 0.29 & 0.04 & 12.33 & 1.92 & 19.19 & 53.43 & 0.16 & 95.93 & \\
\hline $10 / 1$ & 1.56 & 3.88 & 2.60 & 0.28 & 0.10 & 13.56 & 1.68 & 16.78 & 55.29 & 0.30 & 96.10 & \\
\hline $11 / 1$ & 1.58 & 4.24 & 2.54 & 0.20 & 0.08 & 11.99 & 1.92 & 19.20 & 53.75 & 0.24 & 95.79 & \\
\hline $12 / 1$ & 1.56 & 3.64 & 2.60 & 0.30 & 0.00 & 13.80 & 1.63 & 16.27 & 56.44 & 0.25 & 96.49 & \\
\hline $13 / 1$ & 1.25 & 4.67 & 2.49 & 0.15 & 0.10 & 12.13 & 1.92 & 19.19 & 53.75 & 0.21 & 95.95 & \\
\hline $14 / 1$ & 1.50 & 4.00 & 2.58 & 0.23 & 0.08 & 11.80 & 1.85 & 18.54 & 53.92 & 0.19 & 94.69 & \\
\hline $16 / 1$ & 1.67 & 3.88 & 2.56 & 0.33 & 0.12 & 14.12 & 1.65 & 16.51 & 54.82 & 0.30 & 96.00 & \\
\hline $17 / 1$ & 1.51 & 4.27 & 2.81 & 0.19 & 0.09 & 11.40 & 1.95 & 19.53 & 53.37 & 0.16 & 95.34 & \\
\hline $18 / 1$ & 1.36 & 4.50 & 2.77 & 0.28 & 0.11 & 12.69 & 1.86 & 18.59 & 53.58 & 0.21 & 95.99 & \\
\hline $19 / 1$ & 1.53 & 4.18 & 2.68 & 0.38 & 0.08 & 12.99 & 1.78 & 17.79 & 54.83 & 0.22 & 96.45 & \\
\hline $20 / 1$ & 1.66 & 3.37 & 2.68 & 0.27 & 0.09 & 14.03 & 1.68 & 16.84 & 54.29 & 0.59 & 95.56 & $\mathrm{Gl} / 2 \mathrm{a}$ \\
\hline $21 / 1$ & 1.18 & 4.56 & 2.69 & 0.07 & 0.08 & 12.01 & 1.93 & 19.33 & 54.45 & 0.29 & 96.63 & \\
\hline $22 / 1$ & 1.34 & 4.35 & 2.79 & 0.14 & 0.06 & 12.88 & 1.87 & 18.68 & 54.27 & 0.23 & 96.67 & \\
\hline $23 / 1$ & 1.32 & 3.90 & 2.83 & 0.21 & 0.11 & 14.59 & 1.65 & 16.46 & 56.03 & 0.25 & 97.39 & \\
\hline $24 / 1$ & 1.25 & 4.09 & 2.68 & 0.29 & 0.07 & 14.44 & 1.61 & 16.09 & 53.94 & 1.21 & 95.70 & \\
\hline $26 / 1$ & 1.37 & 3.96 & 2.80 & 0.13 & 0.12 & 12.43 & 1.83 & 18.33 & 54.52 & 0.26 & 95.81 & \\
\hline $27 / 1$ & 1.35 & 4.19 & 2.80 & 0.13 & 0.10 & 12.41 & 1.89 & 18.90 & 53.94 & 0.24 & 95.99 & \\
\hline $28 / 1$ & 1.38 & 3.84 & 2.65 & 0.23 & 0.08 & 13.14 & 1.64 & 16.44 & 55.81 & 0.42 & 95.68 & \\
\hline $29 / 1$ & 1.56 & 3.76 & 2.62 & 0.27 & 0.12 & 15.50 & 1.54 & 15.42 & 55.58 & 0.43 & 96.80 & \\
\hline $30 / 1$ & 1.40 & 4.21 & 2.52 & 0.18 & 0.04 & 13.44 & 1.75 & 17.47 & 54.12 & 0.29 & 95.42 & \\
\hline $31 / 1$ & 1.53 & 3.87 & 2.65 & 0.18 & 0.03 & 13.24 & 1.78 & 17.85 & 55.03 & 0.31 & 96.53 & \\
\hline $32 / 1$ & 1.51 & 3.71 & 2.75 & 0.18 & 0.08 & 14.49 & 1.62 & 16.20 & 55.08 & 0.36 & 96.01 & \\
\hline $33 / 1$ & 1.37 & 4.33 & 2.80 & 0.18 & 0.09 & 12.69 & 1.86 & 18.62 & 52.84 & 0.30 & 95.09 & \\
\hline $34 / 1$ & 1.38 & 4.24 & 2.72 & 0.19 & 0.07 & 12.36 & 1.91 & 19.11 & 53.09 & 0.21 & 95.30 & \\
\hline $35 / 1$ & 1.30 & 4.23 & 2.94 & 0.16 & 0.13 & 12.27 & 1.85 & 18.46 & 53.47 & 0.22 & 95.08 & \\
\hline $\begin{array}{l}\text { Mean } \\
\text { (Range of } \\
\text { oxide) }\end{array}$ & $\begin{array}{l}1.47 \\
(1.18- \\
1.72)\end{array}$ & $\begin{array}{c}4.07 \\
(3.18- \\
4.67)\end{array}$ & $\begin{array}{l}2.68 \\
(2.49- \\
2.94)\end{array}$ & $\begin{array}{c}0.23 \\
(0.07- \\
0.38)\end{array}$ & $\begin{array}{l}0.08 \\
(0.0- \\
0.15)\end{array}$ & $\begin{array}{c}13.08 \\
(11.32- \\
15.50)\end{array}$ & $\begin{array}{l}1.78 \\
(1.53- \\
1.99)\end{array}$ & $\begin{array}{c}17.82 \\
(15.31- \\
19.93)\end{array}$ & $\begin{array}{c}54.52 \\
(52.84- \\
56.73)\end{array}$ & $\begin{array}{l}0.32 \\
(0.16- \\
1.21)\end{array}$ & $\begin{array}{c}96.09 \\
(94.69- \\
97.50)\end{array}$ & \\
\hline
\end{tabular}


Table 1. Cont.

\begin{tabular}{|c|c|c|c|c|c|c|c|c|c|c|c|c|}
\hline $\begin{array}{c}\text { DataSet } \\
\text { /Point }\end{array}$ & $\mathrm{Na}_{2} \mathrm{O}$ & $\mathrm{K}_{2} \mathrm{O}$ & $\mathrm{MgO}$ & $\mathrm{CaO}$ & $\mathrm{MnO}$ & $\mathrm{Al}_{2} \mathrm{O}_{3}$ & $\mathrm{FeO}$ & $\mathrm{Fe}_{2} \mathrm{O}_{3}$ & $\mathrm{SiO}_{2}$ & $\mathrm{TiO}_{2}$ & Total & $\begin{array}{c}\text { Sample } \\
\text { No. }\end{array}$ \\
\hline $1 / 1$ & 1.37 & 4.07 & 2.61 & 0.06 & 0.07 & 12.93 & 1.77 & 17.70 & 54.38 & 0.24 & 95.29 & \multirow{33}{*}{$\mathrm{Gl} / 2 \mathrm{~b}$} \\
\hline $3 / 1$ & 1.36 & 4.26 & 2.72 & 0.05 & 0.05 & 12.72 & 1.92 & 19.23 & 52.51 & 0.25 & 95.18 & \\
\hline $4 / 1$ & 1.23 & 3.94 & 2.91 & 0.10 & 0.06 & 13.93 & 1.83 & 18.32 & 54.00 & 0.28 & 96.64 & \\
\hline $5 / 1$ & 1.15 & 5.02 & 2.55 & 0.14 & 0.11 & 10.34 & 2.15 & 21.46 & 52.20 & 0.12 & 95.34 & \\
\hline $6 / 1$ & 1.31 & 4.38 & 2.66 & 0.13 & 0.05 & 15.15 & 1.81 & 18.15 & 52.94 & 0.32 & 97.05 & \\
\hline $7 / 1$ & 1.39 & 4.26 & 2.66 & 0.12 & 0.14 & 12.63 & 1.89 & 18.87 & 52.48 & 0.31 & 94.81 & \\
\hline $8 / 1$ & 1.33 & 4.22 & 2.52 & 0.14 & 0.10 & 12.40 & 1.82 & 18.21 & 54.04 & 0.22 & 95.03 & \\
\hline $9 / 1$ & 1.19 & 4.48 & 2.67 & 0.08 & 0.11 & 11.76 & 2.03 & 20.29 & 53.26 & 0.18 & 96.09 & \\
\hline $10 / 1$ & 1.35 & 3.85 & 2.52 & 0.10 & 0.07 & 13.75 & 1.72 & 17.19 & 55.43 & 0.28 & 96.42 & \\
\hline $12 / 1$ & 1.30 & 4.55 & 2.64 & 0.08 & 0.07 & 11.51 & 1.98 & 19.76 & 53.47 & 0.29 & 95.70 & \\
\hline $13 / 1$ & 1.31 & 4.21 & 2.34 & 0.07 & 0.08 & 12.14 & 1.89 & 18.86 & 54.44 & 0.17 & 95.59 & \\
\hline $14 / 1$ & 1.31 & 4.36 & 2.45 & 0.06 & 0.12 & 13.56 & 1.78 & 17.85 & 54.34 & 0.22 & 96.10 & \\
\hline $15 / 1$ & 1.49 & 3.83 & 2.61 & 0.06 & 0.09 & 13.12 & 1.81 & 18.13 & 54.88 & 0.33 & 96.41 & \\
\hline $16 / 1$ & 1.14 & 4.71 & 2.46 & 0.02 & 0.12 & 11.76 & 1.92 & 19.17 & 53.72 & 0.23 & 95.26 & \\
\hline $17 / 1$ & 1.35 & 3.27 & 2.40 & 0.15 & 0.06 & 15.43 & 1.49 & 14.86 & 55.99 & 0.35 & 95.46 & \\
\hline $18 / 1$ & 1.29 & 4.42 & 2.56 & 0.07 & 0.07 & 11.05 & 2.06 & 20.55 & 53.55 & 0.23 & 95.96 & \\
\hline $19 / 1$ & 1.33 & 3.69 & 2.55 & 0.05 & 0.05 & 12.87 & 1.70 & 16.96 & 54.57 & 0.23 & 94.04 & \\
\hline $20 / 1$ & 1.28 & 4.46 & 2.60 & 0.05 & 0.07 & 12.33 & 1.92 & 19.17 & 52.73 & 0.24 & 94.88 & \\
\hline $21 / 1$ & 1.39 & 4.19 & 2.51 & 0.06 & 0.10 & 12.32 & 1.83 & 18.29 & 54.33 & 0.38 & 95.47 & \\
\hline $22 / 1$ & 1.19 & 4.25 & 2.45 & 0.12 & 0.04 & 11.35 & 2.01 & 20.10 & 53.60 & 0.21 & 95.45 & \\
\hline $23 / 1$ & 1.16 & 4.96 & 2.58 & 0.10 & 0.18 & 9.87 & 2.20 & 22.02 & 52.11 & 0.25 & 95.52 & \\
\hline $25 / 1$ & 1.40 & 4.39 & 2.38 & 0.06 & 0.12 & 12.88 & 2.01 & 20.08 & 52.89 & 0.20 & 96.47 & \\
\hline $26 / 1$ & 1.10 & 4.47 & 2.49 & 0.07 & 0.14 & 11.59 & 1.94 & 19.37 & 54.55 & 0.23 & 96.01 & \\
\hline $27 / 1$ & 1.46 & 3.85 & 2.48 & 0.12 & 0.16 & 15.58 & 1.74 & 17.37 & 57.20 & 0.27 & 100.29 & \\
\hline $28 / 1$ & 1.36 & 3.96 & 2.50 & 0.07 & 0.14 & 13.71 & 1.68 & 16.82 & 54.08 & 0.27 & 94.70 & \\
\hline $29 / 1$ & 1.10 & 4.54 & 2.45 & 0.04 & 0.19 & 12.60 & 1.89 & 18.91 & 53.77 & 0.26 & 95.77 & \\
\hline $31 / 1$ & 1.20 & 4.50 & 2.36 & 0.08 & 0.12 & 11.49 & 1.92 & 19.22 & 54.41 & 0.28 & 95.65 & \\
\hline $33 / 1$ & 1.24 & 4.29 & 2.51 & 0.06 & 0.22 & 12.29 & 1.87 & 18.67 & 54.84 & 0.23 & 96.34 & \\
\hline $34 / 1$ & 1.23 & 3.49 & 2.06 & 0.09 & 0.17 & 13.79 & 1.60 & 15.96 & 51.72 & 0.40 & 90.64 & \\
\hline $35 / 1$ & 1.11 & 4.74 & 2.41 & 0.07 & 0.18 & 11.07 & 2.11 & 21.14 & 53.09 & 0.26 & 96.33 & \\
\hline $38 / 1$ & 1.09 & 4.19 & 2.38 & 0.03 & 0.07 & 15.55 & 1.64 & 16.39 & 52.69 & 0.49 & 94.66 & \\
\hline $39 / 1$ & 1.24 & 4.33 & 2.50 & 0.01 & 0.11 & 12.92 & 1.81 & 18.11 & 54.29 & 0.41 & 95.78 & \\
\hline $\begin{array}{c}\text { Mean } \\
\text { (Range of } \\
\text { oxide) }\end{array}$ & $\begin{array}{l}1.27 \\
(1.09- \\
1.49)\end{array}$ & $\begin{array}{l}4.25 \\
(3.27- \\
5.02)\end{array}$ & $\begin{array}{l}2.51 \\
(2.06- \\
2.91)\end{array}$ & $\begin{array}{l}0.08 \\
(0.01- \\
0.15)\end{array}$ & $\begin{array}{c}0.11 \\
(0.04- \\
0.22)\end{array}$ & $\begin{array}{l}12.70 \\
(9.87- \\
15.58)\end{array}$ & $\begin{array}{l}1.87 \\
(1.49- \\
2.20)\end{array}$ & $\begin{array}{c}18.66 \\
(14.86- \\
22.02)\end{array}$ & $\begin{array}{c}53.83 \\
(51.72- \\
57.20)\end{array}$ & $\begin{array}{c}0.27 \\
(0.12- \\
0.49)\end{array}$ & $\begin{array}{c}95.63 \\
(90.64- \\
100.29)\end{array}$ & \\
\hline $1 / 1$ & 1.34 & 4.57 & 2.50 & 0.14 & 0.09 & 12.64 & 1.85 & 18.52 & 51.61 & 0.24 & 93.57 & \multirow{28}{*}{$\mathrm{Gl} / 2 \mathrm{c}$} \\
\hline $2 / 1$ & 1.59 & 3.49 & 2.49 & 0.26 & 0.04 & 15.21 & 1.58 & 15.77 & 53.59 & 0.30 & 94.41 & \\
\hline $4 / 1$ & 1.77 & 3.80 & 2.86 & 0.26 & 0.06 & 13.77 & 1.79 & 17.88 & 55.44 & 0.22 & 97.90 & \\
\hline $6 / 1$ & 1.53 & 3.88 & 2.58 & 0.21 & 0.07 & 14.00 & 1.78 & 17.76 & 53.74 & 0.28 & 95.89 & \\
\hline $7 / 1$ & 1.55 & 4.13 & 2.52 & 0.09 & 0.08 & 14.74 & 1.69 & 16.91 & 53.56 & 0.37 & 95.76 & \\
\hline $8 / 1$ & 1.53 & 4.42 & 2.58 & 0.23 & 0.09 & 11.83 & 1.91 & 19.10 & 53.38 & 0.26 & 95.45 & \\
\hline $9 / 1$ & 1.42 & 4.81 & 2.76 & 0.20 & 0.08 & 11.09 & 2.18 & 21.78 & 51.45 & 0.23 & 96.05 & \\
\hline $10 / 1$ & 1.72 & 3.95 & 2.64 & 0.12 & 0.04 & 13.38 & 1.77 & 17.74 & 55.34 & 0.32 & 97.05 & \\
\hline $11 / 1$ & 1.48 & 3.62 & 2.53 & 0.15 & 0.07 & 16.77 & 1.55 & 15.52 & 54.06 & 0.29 & 96.09 & \\
\hline $12 / 1$ & 1.66 & 3.87 & 2.63 & 0.15 & 0.08 & 12.89 & 1.82 & 18.24 & 54.15 & 0.27 & 95.90 & \\
\hline $13 / 1$ & 1.34 & 4.55 & 2.58 & 0.08 & 0.11 & 12.17 & 1.88 & 18.79 & 54.02 & 0.25 & 95.88 & \\
\hline $14 / 1$ & 1.82 & 4.30 & 2.69 & 0.13 & 0.11 & 13.66 & 1.77 & 17.73 & 53.89 & 0.23 & 96.42 & \\
\hline $15 / 1$ & 1.82 & 4.12 & 2.50 & 0.08 & 0.10 & 13.32 & 1.70 & 17.04 & 55.17 & 0.27 & 96.16 & \\
\hline $17 / 1$ & 1.60 & 4.12 & 2.65 & 0.10 & 0.09 & 12.57 & 1.89 & 18.91 & 53.74 & 0.26 & 95.98 & \\
\hline $19 / 1$ & 1.62 & 4.14 & 2.61 & 0.12 & 0.07 & 11.34 & 1.84 & 18.43 & 52.54 & 0.24 & 93.02 & \\
\hline $21 / 1$ & 1.73 & 3.81 & 2.73 & 0.10 & 0.07 & 13.74 & 1.69 & 16.95 & 55.25 & 0.29 & 96.44 & \\
\hline $22 / 1$ & 1.90 & 3.84 & 2.66 & 0.10 & 0.08 & 13.62 & 1.71 & 17.11 & 54.71 & 0.28 & 96.13 & \\
\hline $24 / 1$ & 1.49 & 4.30 & 2.55 & 0.12 & 0.11 & 12.81 & 1.87 & 18.73 & 51.95 & 0.33 & 94.32 & \\
\hline $27 / 1$ & 1.53 & 3.44 & 2.58 & 0.07 & 0.06 & 15.45 & 1.53 & 15.30 & 55.78 & 0.74 & 96.50 & \\
\hline $28 / 1$ & 1.63 & 3.35 & 2.52 & 0.15 & 0.07 & 14.73 & 1.56 & 15.61 & 55.23 & 0.41 & 95.29 & \\
\hline $29 / 1$ & 1.73 & 4.01 & 2.74 & 0.11 & 0.06 & 12.92 & 1.82 & 18.20 & 54.05 & 0.31 & 95.98 & \\
\hline $30 / 1$ & 1.64 & 4.12 & 2.69 & 0.16 & 0.08 & 12.82 & 1.89 & 18.87 & 53.83 & 0.25 & 96.35 & \\
\hline $31 / 1$ & 1.75 & 3.51 & 2.51 & 0.13 & 0.08 & 14.73 & 1.56 & 15.60 & 56.87 & 0.24 & 97.03 & \\
\hline $32 / 1$ & 1.47 & 4.33 & 2.53 & 0.14 & 0.06 & 12.41 & 1.88 & 18.79 & 53.52 & 0.26 & 95.46 & \\
\hline $33 / 1$ & 1.56 & 3.69 & 2.58 & 0.09 & 0.10 & 14.40 & 1.65 & 16.45 & 56.57 & 0.25 & 97.38 & \\
\hline $34 / 1$ & 1.72 & 4.05 & 2.54 & 0.13 & 0.07 & 13.12 & 1.84 & 18.43 & 53.21 & 0.33 & 95.48 & \\
\hline $35 / 1$ & 1.48 & 4.34 & 2.55 & 0.09 & 0.09 & 13.06 & 1.83 & 18.29 & 54.43 & 0.31 & 96.49 & \\
\hline $36 / 1$ & 1.27 & 4.34 & 2.53 & 0.02 & 0.08 & 14.19 & 1.77 & 17.66 & 54.21 & 0.35 & 96.45 & \\
\hline
\end{tabular}


Table 1. Cont.

\begin{tabular}{|c|c|c|c|c|c|c|c|c|c|c|c|c|}
\hline $\begin{array}{l}\text { DataSet } \\
\text { /Point }\end{array}$ & $\mathrm{Na}_{2} \mathrm{O}$ & $\mathrm{K}_{2} \mathrm{O}$ & MgO & $\mathrm{CaO}$ & $\mathrm{MnO}$ & $\mathrm{Al}_{2} \mathrm{O}_{3}$ & $\mathrm{FeO}$ & $\mathrm{Fe}_{2} \mathrm{O}_{3}$ & $\mathrm{SiO}_{2}$ & $\mathrm{TiO}_{2}$ & Total & $\begin{array}{c}\text { Sample } \\
\text { No. }\end{array}$ \\
\hline $38 / 1$ & 1.54 & 4.33 & 2.54 & 0.11 & 0.14 & 12.32 & 1.98 & 19.76 & 52.23 & 0.41 & 95.40 & \\
\hline $39 / 1$ & 1.67 & 3.49 & 2.62 & 0.16 & 0.07 & 13.44 & 1.73 & 17.31 & 53.91 & 0.30 & 94.70 & \\
\hline $40 / 1$ & 1.63 & 4.15 & 2.44 & 0.16 & 0.05 & 12.42 & 1.78 & 17.81 & 53.65 & 0.26 & 94.36 & \\
\hline $41 / 1$ & 1.81 & 3.90 & 2.66 & 0.16 & 0.08 & 13.93 & 1.75 & 17.50 & 54.12 & 0.30 & 96.27 & \\
\hline $44 / 1$ & 1.54 & 4.45 & 2.67 & 0.09 & 0.09 & 11.48 & 1.92 & 19.16 & 52.13 & 0.20 & 93.74 & \\
\hline $45 / 1$ & 1.73 & 3.29 & 2.53 & 0.20 & 0.12 & 13.45 & 1.63 & 16.32 & 52.27 & 3.98 & 95.56 & \\
\hline $46 / 1$ & 1.60 & 4.14 & 2.69 & 0.24 & 0.07 & 13.72 & 1.80 & 18.03 & 53.49 & 0.32 & 96.20 & \\
\hline $47 / 1$ & 1.78 & 3.87 & 2.53 & 0.14 & 0.06 & 14.61 & 1.71 & 17.14 & 54.29 & 0.28 & 96.51 & \\
\hline $48 / 1$ & 1.61 & 4.15 & 2.78 & 0.17 & 0.15 & 12.25 & 1.90 & 19.00 & 52.86 & 0.26 & 95.21 & \\
\hline $\begin{array}{c}\text { Mean } \\
\text { (Range of } \\
\text { oxide) }\end{array}$ & $\begin{array}{l}1.61 \\
(1.27- \\
1.90)\end{array}$ & $\begin{array}{c}4.02 \\
(3.29- \\
4.81)\end{array}$ & $\begin{array}{l}2.60 \\
(2.44- \\
2.86)\end{array}$ & $\begin{array}{c}0.14 \\
(0.02- \\
0.26)\end{array}$ & $\begin{array}{c}0.08 \\
(0.04- \\
0.15)\end{array}$ & $\begin{array}{c}13.38 \\
(11.09- \\
16.77)\end{array}$ & $\begin{array}{c}1.78 \\
(1.53- \\
2.18)\end{array}$ & $\begin{array}{c}17.79 \\
(15.30- \\
21.78)\end{array}$ & $\begin{array}{c}53.90 \\
(51.45- \\
56.87)\end{array}$ & $\begin{array}{c}0.40 \\
(0.20- \\
3.98)\end{array}$ & $\begin{array}{c}95.75 \\
(93.02- \\
97.90)\end{array}$ & \\
\hline $2 / 1$ & 0.72 & 4.67 & 2.65 & 0.11 & 0.16 & 10.94 & 2.08 & 20.79 & 53.30 & 0.21 & 95.63 & \\
\hline $3 / 1$ & 0.87 & 3.77 & 2.68 & 0.16 & 0.04 & 14.70 & 1.56 & 15.65 & 56.17 & 0.32 & 95.93 & \\
\hline $4 / 1$ & 1.00 & 3.79 & 3.04 & 0.37 & 0.08 & 14.51 & 1.76 & 17.60 & 53.35 & 0.32 & 95.84 & \\
\hline $6 / 1$ & 0.99 & 4.02 & 3.04 & 0.26 & 0.16 & 12.30 & 1.89 & 18.92 & 55.15 & 0.34 & 97.09 & \\
\hline $7 / 1$ & 1.11 & 4.02 & 2.54 & 0.12 & 0.13 & 14.69 & 1.73 & 17.30 & 53.92 & 0.31 & 95.89 & \\
\hline $8 / 1$ & 0.64 & 5.19 & 2.78 & 0.57 & 0.17 & 11.28 & 2.00 & 20.00 & 52.25 & 0.26 & 95.20 & \\
\hline $9 / 1$ & 1.23 & 4.11 & 2.89 & 0.48 & 0.05 & 13.97 & 1.68 & 16.80 & 54.21 & 0.29 & 95.77 & \\
\hline $10 / 1$ & 0.88 & 4.76 & 2.84 & 0.50 & 0.16 & 10.91 & 2.06 & 20.64 & 52.71 & 0.19 & 95.66 & \\
\hline $11 / 1$ & 0.79 & 5.52 & 2.44 & 0.69 & 0.21 & 14.80 & 1.65 & 16.49 & 51.84 & 0.23 & 94.66 & \\
\hline $13 / 1$ & 1.01 & 4.28 & 2.69 & 0.49 & 0.16 & 13.60 & 1.76 & 17.61 & 54.42 & 0.36 & 96.43 & \\
\hline $14 / 1$ & 1.05 & 4.50 & 2.78 & 0.67 & 0.12 & 13.70 & 1.82 & 18.23 & 53.13 & 0.27 & 96.28 & \\
\hline $16 / 1$ & 0.82 & 4.87 & 2.76 & 0.45 & 0.13 & 11.01 & 2.11 & 21.07 & 50.64 & 0.25 & 94.10 & \\
\hline $18 / 1$ & 0.60 & 4.17 & 2.97 & 0.32 & 0.14 & 10.89 & 1.83 & 18.28 & 48.48 & 0.30 & 88.02 & \\
\hline $19 / 1$ & 1.71 & 4.08 & 2.94 & 0.37 & 0.08 & 13.57 & 1.72 & 17.18 & 54.39 & 0.31 & 96.36 & $\mathrm{Gl} / 2 \mathrm{~d}$ \\
\hline $20 / 1$ & 0.31 & 4.62 & 2.61 & 0.24 & 0.16 & 11.83 & 1.87 & 18.72 & 51.61 & 0.30 & 92.52 & \\
\hline $22 / 1$ & 0.47 & 5.29 & 2.90 & 0.92 & 0.25 & 10.62 & 2.06 & 20.60 & 52.82 & 0.17 & 96.11 & \\
\hline $23 / 1$ & 1.41 & 3.09 & 4.47 & 0.66 & 0.22 & 13.22 & 1.52 & 15.17 & 48.96 & 0.17 & 88.89 & \\
\hline $28 / 1$ & 0.48 & 4.09 & 3.80 & 0.90 & 0.38 & 14.28 & 1.52 & 15.23 & 53.81 & 0.31 & 94.83 & \\
\hline $29 / 1$ & 0.90 & 4.56 & 2.91 & 0.54 & 0.12 & 12.59 & 1.88 & 18.83 & 52.90 & 0.24 & 95.47 & \\
\hline $30 / 1$ & 0.96 & 3.31 & 2.91 & 0.46 & 0.11 & 16.67 & 1.48 & 14.81 & 55.00 & 0.39 & 96.16 & \\
\hline $33 / 1$ & 0.24 & 4.10 & 2.60 & 0.31 & 0.05 & 10.10 & 2.12 & 21.16 & 50.11 & 0.15 & 90.95 & \\
\hline $34 / 1$ & 0.22 & 3.51 & 2.92 & 0.65 & 0.09 & 11.27 & 1.90 & 19.01 & 49.48 & 0.32 & 89.38 & \\
\hline $35 / 1$ & 0.54 & 4.06 & 2.80 & 0.58 & 0.13 & 13.71 & 1.73 & 17.26 & 49.08 & 0.30 & 90.21 & \\
\hline $36 / 1$ & 0.64 & 3.99 & 2.84 & 0.70 & 0.13 & 12.40 & 1.77 & 17.66 & 52.57 & 0.21 & 92.95 & \\
\hline $37 / 1$ & 0.58 & 3.93 & 2.89 & 0.93 & 0.11 & 13.83 & 1.63 & 16.32 & 52.17 & 0.25 & 92.68 & \\
\hline $\begin{array}{c}\text { Mean } \\
\text { (Range of } \\
\text { oxide) }\end{array}$ & $\begin{array}{l}0.81 \\
(0.22- \\
1.70)\end{array}$ & $\begin{array}{c}4.25 \\
(3.09- \\
5.52)\end{array}$ & $\begin{array}{c}2.91 \\
(2.44- \\
4.47)\end{array}$ & $\begin{array}{c}0.50 \\
(0.11- \\
0.93)\end{array}$ & $\begin{array}{c}0.14 \\
(0.04- \\
0.38)\end{array}$ & $\begin{array}{c}12.85 \\
(10.10- \\
16.67)\end{array}$ & $\begin{array}{c}1.81 \\
(1.48- \\
2.12)\end{array}$ & $\begin{array}{c}18.05 \\
(14.81- \\
21.16)\end{array}$ & $\begin{array}{c}52.50 \\
(48.48- \\
56.17)\end{array}$ & $\begin{array}{c}0.27 \\
(0.15- \\
0.39)\end{array}$ & $\begin{array}{c}94.12 \\
(88.02- \\
97.09)\end{array}$ & \\
\hline
\end{tabular}

All EPMA data were normalized to $100 \mathrm{wt} \%$ on an anhydrous basis for different cross-plots. The cross-plot between $\mathrm{K}_{2} \mathrm{O}$ and $\mathrm{Fe}_{2} \mathrm{O}_{3}$ (total) exhibits a moderate positive correlation $\left(r^{2}=0.6\right)$. $\mathrm{Na}_{2} \mathrm{O}$ manifest poor negative correlation with $\mathrm{Fe}_{2} \mathrm{O}_{3}$ (total) (Figure 4a). The $\mathrm{Al}_{2} \mathrm{O}_{3}$ versus $\mathrm{Fe}_{2} \mathrm{O}_{3}$ (total) cross-plot exhibits a good negative correlation $\left(r^{2}=0.8\right)$ (Figure $4 \mathrm{~B})$. The $\mathrm{K}_{2} \mathrm{O}$ vs. $\mathrm{Al}_{2} \mathrm{O}_{3}$ plot indicates a moderate negative correlation $\left(r^{2}=0.5\right)$. On the other hand, the $\mathrm{Na}_{2} \mathrm{O}$ vs. $\mathrm{Al}_{2} \mathrm{O}_{3}$ cross-plot shows a poor positive correlation (Figure $4 \mathrm{C}$ ). The $\mathrm{K}_{2} \mathrm{O}$ vs. $\mathrm{Na}_{2} \mathrm{O}$ cross-plot shows a poor negative correlation (Figure $4 \mathrm{D}$ ). 


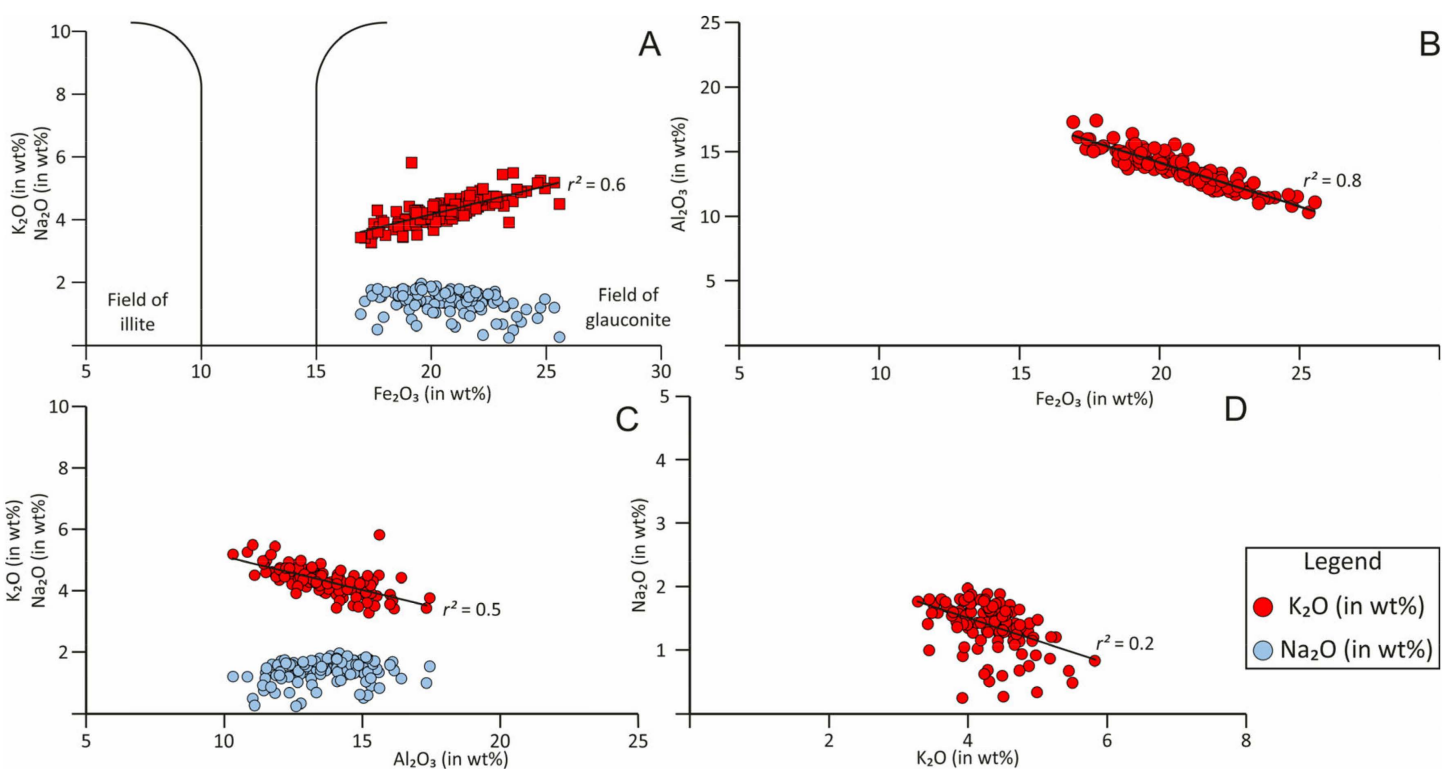

Figure 4. Cross-plots of $\mathrm{K}_{2} \mathrm{O}$ and $\mathrm{Na}_{2} \mathrm{O}$ vs. $\mathrm{Fe}_{2} \mathrm{O}_{3}(\mathbf{A}), \mathrm{Al}_{2} \mathrm{O}_{3}$ vs. $\mathrm{Fe}_{2} \mathrm{O}_{3}(\mathbf{B}), \mathrm{K}_{2} \mathrm{O}$ and $\mathrm{Na}_{2} \mathrm{O}$ vs. $\mathrm{Al}_{2} \mathrm{O}_{3}$ (C), and $\mathrm{K}_{2} \mathrm{O}$ vs. $\mathrm{Na}_{2} \mathrm{O}$ (D) (fields of glauconite and ferric illite are after Odin and Matter [1]). Note that the interlayer cations $\mathrm{K}_{2} \mathrm{O}$ and $\mathrm{Na}_{2} \mathrm{O}$ show an opposite trend.

Bi-variant plots involving $\mathrm{K}_{2} \mathrm{O}$ and $\mathrm{Na}_{2} \mathrm{O}, \mathrm{Fe}_{2} \mathrm{O}_{3}$ (total) and $\mathrm{Al}_{2} \mathrm{O}_{3}$ reflect the chemical pathways of glauconite maturation. The positive correlation between $\mathrm{K}_{2} \mathrm{O}$ and $\mathrm{Fe}_{2} \mathrm{O}_{3}$ (total) (Figure $4 \mathrm{~A}$ ) indicates simultaneous intake of $\mathrm{K}$ and $\mathrm{Fe}$ in the glauconite structure. The negative correlation between $\mathrm{K}_{2} \mathrm{O}$ and $\mathrm{Na}_{2} \mathrm{O}$ indicates that $\mathrm{Na}$ fixation into the glauconite structure is accompanied by the expulsion of $\mathrm{K}$ from the interlayer sites (Figure $4 \mathrm{D}$ ). The good negative correlation between $\mathrm{Al}_{2} \mathrm{O}_{3}$ and $\mathrm{Fe}_{2} \mathrm{O}_{3}$ (total) (Figure $4 \mathrm{C}$ ) indicates the substitution of $\mathrm{Al}$ by $\mathrm{Fe}$ in the octahedral site during the glauconitization $[1,2,17,40]$. Therefore, the negative correlation of $\mathrm{K}_{2} \mathrm{O}$ and $\mathrm{Al}_{2} \mathrm{O}_{3}$ (Figure $4 \mathrm{C}$ ) implies the addition of $\mathrm{K}^{+}$accompanied by the expulsion of $\mathrm{Al}^{3+}$ from the glauconite structure. Iron is mobile in the $\mathrm{Fe}^{2+}$ state, which replaces the $\mathrm{Al}^{3+}$ in the octahedral site and creates a charge imbalance in the glauconite structure. The $\mathrm{K}^{+}$is incorporated in the interlayer sites of the glauconite structure for charge balancing, which causes the negative correlation between $\mathrm{K}_{2} \mathrm{O}$ and $\mathrm{Al}_{2} \mathrm{O}_{3}$.

\subsection{Mineralogy and Micro-Textural Study of Glauconite}

The X-ray diffraction analysis of the randomly oriented clay fraction $(<2 \mu \mathrm{m})$ exhibits broad, asymmetric peaks of lower diffraction angles $\left(<10^{\circ} 2 \theta\right)$ (Figure 5$)$. Other characteristic peaks include asymmetric peaks at $4.48 \AA, 3.25 \AA, 2.57 \AA, 1.97 \AA$, and $1.51 \AA$ (Figure 5). The clay fraction also shows a few sharp and symmetric reflections at $4.26 \AA, 3.61 \AA, 3.35 \AA$, and $2.81 \AA$ along with a few less intense but sharp and symmetric peaks at $2.46 \AA, 2.36 \AA$, $2.28 \AA, 2.24 \AA, 2.14 \AA, 1.81 \AA, 1.74 \AA$, and $1.54 \AA$. The X-ray diffractogram of smear-mounted, oriented glauconite grains exhibits asymmetric (00l) reflections with a broad base (Figure 5). The air-dried sample shows (001) reflection at $11.89 \AA$. The (002) and (004) reflections are subdued and are positioned at $5.01 \AA$ and $2.55 \AA$, respectively. The (003) reflection appears at $3.24 \AA$ with a broad base and less asymmetrical peak. Upon glycolation, the basal (001) peak expands to $11.89 \AA$ A with a broad hump (Figure 5). Peaks corresponding to (002) and (004) reflections show a slight shift to $5.10 \AA$ and $2.62 \AA$, respectively. The (003) peak shifts to $3.31 \AA$ after the glycolation. All the peaks widen upon glycolation. The heated sample $\left(550^{\circ} \mathrm{C}\right.$ for $1 \mathrm{~h}$ ) shows symmetric and sharp basal reflection. The (001) peak collapses to $9.78 \AA$ while (002) and (004) reflections appear at $4.90 \AA$ and $2.50 \AA$, respectively, after heating. The (003) reflection shifts to $3.27 \AA$ and appears symmetric and sharp. A minor peak, appearing at $7.07 \AA$ in air-dried and glycolated samples, disappears after heating (Figure 5). 


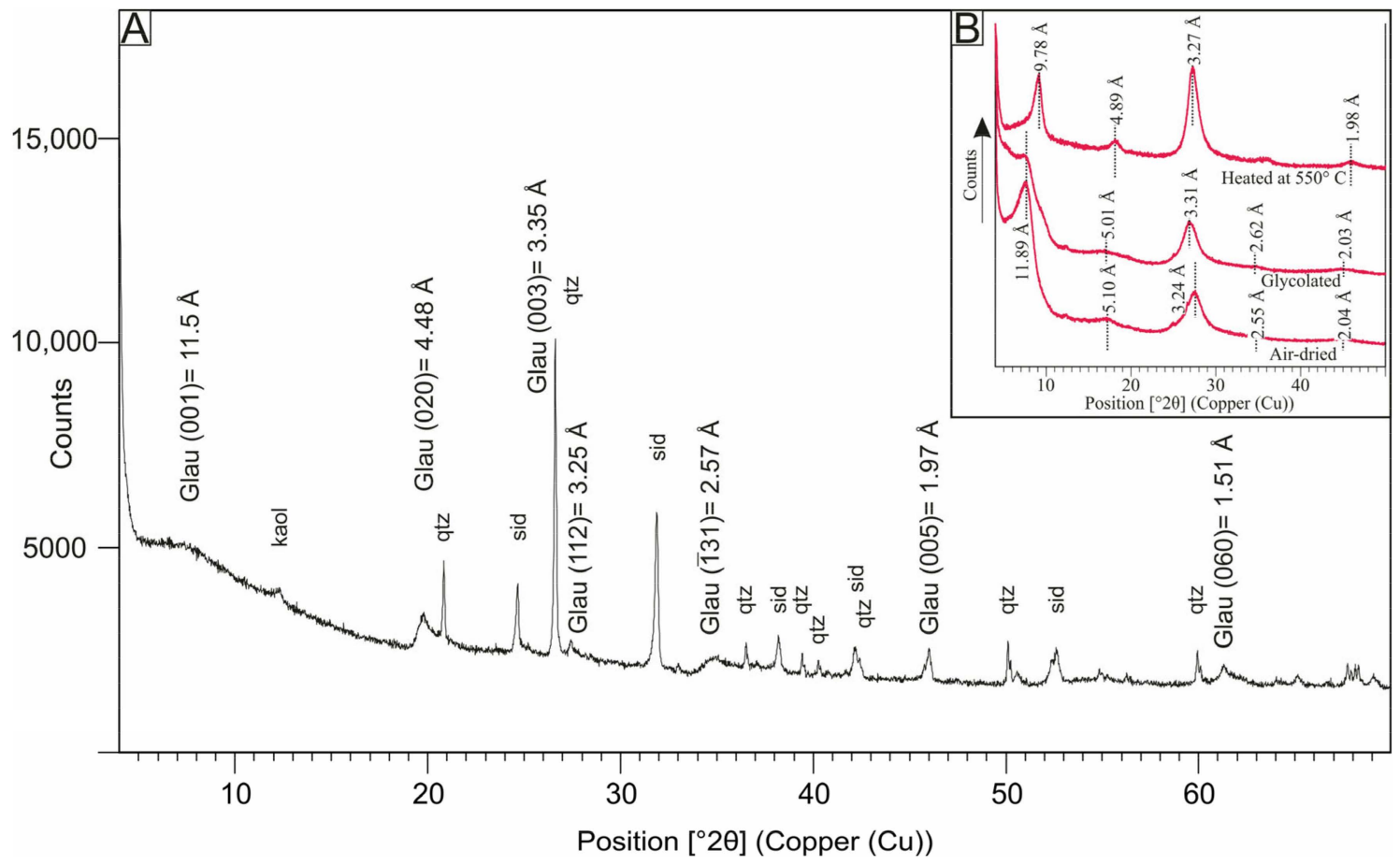

Figure 5. (A) X-ray diffractogram of randomly oriented clay fraction ( $<2 \mu \mathrm{m}$ grain size) exhibiting glauconite and kaolinite as dominant phyllosilicate phases with minor siderite and quartz impurities. (B) X-ray diffraction pattern of oriented smear-mounted glauconite pellets exhibiting basal (00l) reflections. Glau = glauconite, $\mathrm{kaol}=$ kaolinite, $\mathrm{qtz}=$ quartz , and sid = siderite.

The peaks at $4.48 \AA, 3.25 \AA, 2.57 \AA, 1.97 \AA$, and $1.51 \AA$ along with basal (001) reflection at $11.5 \AA$ in randomly oriented clay fraction characterize both glauconite and illite (Figure 5). The basal (001) reflection of glauconite appears within $10 \AA$ and $14 \AA$. The ordered glauconite has a $10 \AA$ basal spacing while the smectite minerals exhibit $14 \AA$ A basal spacing $[1,12-15,43]$. The basal reflection of the (001) plane at $11.89 \AA$ in air-dried Giral samples expands upon glycolation, indicating considerable smectite interstratification within glauconite [44]. The high value of the (001) peak is consistent with 'nascent' to 'slightly evolved' glauconite [45]. The subdued (002) and (004) reflections distinguish the glauconitic minerals in Giral samples from illite and indicate the high octahedral iron [43,46-48]. The minor peak at $7.07 \AA$ in air-dried and glycolated samples, which disappears after heating at $550{ }^{\circ} \mathrm{C}$, mark the presence of kaolinite $[47,48]$. The sharp and symmetrical reflections at $2.81 \AA, 2.14 \AA$, and $1.74 \AA$ and at $4.26 \AA, 3.35 \AA, 1.81 \AA$, and $1.54 \AA$, indicate siderite and quartz, respectively.

High-resolution micro-textural study of the glauconites reveals tiny, blade-like particles, which are often curled. This texture is typical for immature, slightly evolved glauconite. In places, tiny, sheet-like structures (marked by the yellow arrow) of kaolinite transform into curved flakes of slightly evolved glauconites (Figure 6A-D). 

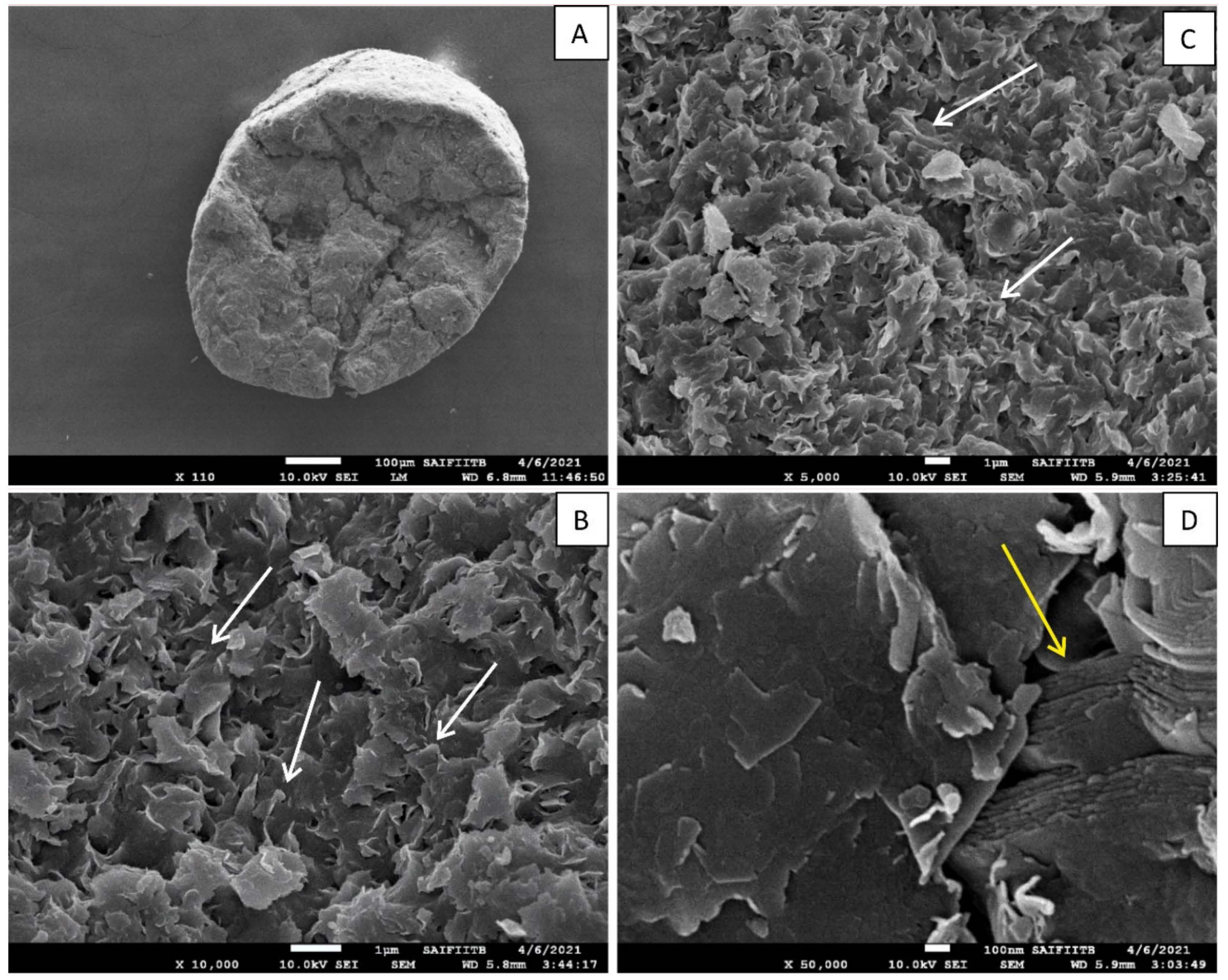

Figure 6. FEG-SEM image showing the micro-texture of the glauconite showing radial fractures within the glauconite pellets (A). Pellets showing tiny, blade-like structures (white arrows) reflecting 'slightly evolved' glauconite (B,C). Sheet-like structures of kaolinite (yellow arrow) (D).

\subsection{Mössbauer Spectroscopy and Structural Formula of Glauconite}

Mössbauer spectroscopic analysis of glauconite pellets reveals five doublets (Table 2; Figure 7). Doublets $\mathrm{A}, \mathrm{B}$, and $\mathrm{C}$ show low isomer shift $(\delta)$ values ranging between $\delta=0.319-0.405 \mathrm{~mm} / \mathrm{s}$. Doublets $\mathrm{D}$ and $\mathrm{E}$ show relatively higher isomer shift $(\delta)$ values, ranging between $\delta=1.120-1.150 \mathrm{~mm} / \mathrm{s}$. The quadrupole splitting $\left(\Delta E_{Q}\right)$ values of doublets $\mathrm{A}, \mathrm{B}$, and $\mathrm{C}$ are lower than that of doublets $\mathrm{D}$ and $\mathrm{E}$. Quadrupole splitting $\left(\Delta E_{Q}\right)$ of doublets A, B, and C vary between $0.363-1.243 \mathrm{~mm} / \mathrm{s}$. Doublets B and A show the least and the highest quadrupole splitting values, respectively. Doublets $\mathrm{D}$ and $\mathrm{E}$ are characterized by high quadrupole splitting with $\Delta E_{Q}$ ranging from 2.193 to $2.884 \mathrm{~mm} / \mathrm{s}$.

The Mössbauer spectra of glauconite manifest five doublets indicating octahedral $\mathrm{Al}$ for Fe substitution [49-51]. Iron is represented in both ferric $\left(\mathrm{Fe}^{3+}\right)$ and ferrous $\left(\mathrm{Fe}^{2+}\right)$ states. The small isomer shifts of doublets $\mathrm{A}, \mathrm{B}$, and $\mathrm{C}$ correspond to $\mathrm{Fe}^{3+}$ in a high-spin state while the large isomer shifts of doublets $\mathrm{D}$ and $\mathrm{E}$ correspond to $\mathrm{Fe}^{2+}$. The small quadrupole splitting of doublet $\mathrm{B}$ corresponds to $\mathrm{Fe}^{3+}$ in the less distorted, symmetrical cis $\mathrm{M}$ (2). $\mathrm{Fe}^{3+}$ also occupies the larger, trans-M (1) site, which is attested by the large quadrupole splitting of doublets $\mathrm{A}$ and $\mathrm{C}$. Doublets D and $\mathrm{E}$ with very large quadrupole splitting correlate to $\mathrm{Fe}^{2+}$ in the trans-M (1) site. 
Table 2. Mössbauer spectral parameters of Giral glauconite extracted from analysis of Mössbauer spectra recorded at room temperature.

\begin{tabular}{|c|c|c|c|c|c|c|c|}
\hline Sample & $\begin{array}{l}\text { Iron Sites } \\
\text { (Doublets) }\end{array}$ & $\begin{array}{c}\text { Isomer Shift ( } \delta \text { ) } \\
\mathrm{mm} / \mathrm{s} \pm 0.005\end{array}$ & $\begin{array}{l}\text { Quadrupole } \\
\text { Splitting }(\Delta) \\
\mathrm{mm} / \mathrm{s} \pm 0.01\end{array}$ & $\begin{array}{c}\text { Line Width }(\Gamma) \\
\mathrm{mm} / \mathrm{s} \pm 0.04\end{array}$ & $\begin{array}{c}\text { Relative } \\
\text { Area } \\
\left(R_{\mathrm{A}}\right) \\
\%\end{array}$ & $\begin{array}{c}\text { Ratio } \\
\mathrm{Fe}^{2+} / \mathrm{Fe}^{3+}\end{array}$ & $\begin{array}{c}\text { Fitting } \\
\text { Quality }\left(\chi^{2}\right)\end{array}$ \\
\hline \multirow{5}{*}{ GL-18-2 } & $\begin{array}{c}\text { Doublet } \mathrm{A}\left(\mathrm{Fe}^{3+}\right) \\
\text { trans } \mathrm{M}(1)\end{array}$ & 0.405 & 1.243 & 0.406 & 13.7 & \multirow{5}{*}{0.10} & \multirow{5}{*}{1.03} \\
\hline & $\begin{array}{l}\text { Doublet } \mathrm{B}\left(\mathrm{Fe}^{3+}\right) \\
\text { cis } \mathrm{m}^{2}\end{array}$ & 0.343 & 0.363 & 0.479 & 60.7 & & \\
\hline & $\begin{array}{c}\text { Doublet } \mathrm{C}\left(\mathrm{Fe}^{3+}\right) \\
\text { trans } \mathrm{M}(1)\end{array}$ & 0.319 & 0.871 & 0.386 & 16.2 & & \\
\hline & $\begin{array}{c}\text { Doublet } \mathrm{D}\left(\mathrm{Fe}^{2+}\right) \\
\text { trans } \mathrm{M}(1)\end{array}$ & 1.150 & 2.884 & 0.35 & 5.0 & & \\
\hline & $\begin{array}{l}\text { Doublet } \mathrm{E}\left(\mathrm{Fe}^{2+}\right) \\
\text { trans } \mathrm{M}(1)\end{array}$ & 1.120 & 2.193 & 0.350 & 4.4 & & \\
\hline
\end{tabular}

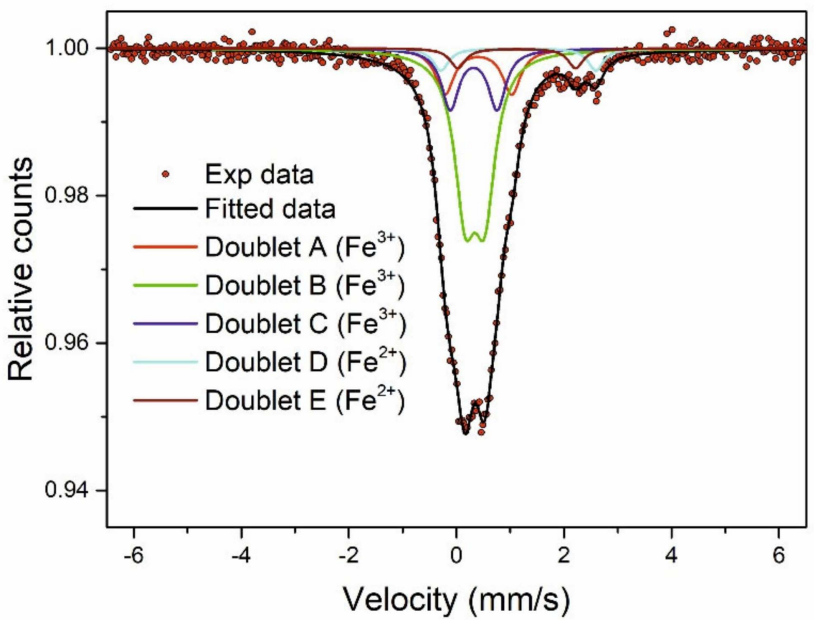

Figure 7. Representative Mössbauer spectrum of glauconite pellet of Dharvi Dungar Formation fitted with five doublets representing octahedral occupancy by both $\mathrm{Fe}^{2+}$ and $\mathrm{Fe}^{3+}$ ions.

The $\mathrm{Fe}^{2+} / \mathrm{Fe}^{3+}$ ratio of the glauconite was calculated using the relative area (under the curve in Figure 7) of the $\mathrm{Fe}^{2+}$ and $\mathrm{Fe}^{3+}$ cations by taking into consideration the $\chi^{2}$ value (fitting parameter) obtained from the Mössbauer spectra. The calculated $\mathrm{Fe}^{2+} / \mathrm{Fe}^{3+}$ ratio of 0.10 fairly agrees with Phanerozoic glauconites $[3,19,52,53]$. This ratio is used to calculate the $\mathrm{wt} \%$ equivalent of $\mathrm{FeO}$ and $\mathrm{Fe}_{2} \mathrm{O}_{3}$ to obtain the chemical formula of glauconite. The average chemical formula of glauconite of the Giral Member is as follows:

$$
\left(\mathrm{K}_{0.36} \mathrm{Na}_{0.18} \mathrm{Ca}_{0.00}\right)_{0.54}\left(\mathrm{Al}_{0.73} \mathrm{Fe}^{3+}{ }_{0.93} \mathrm{Fe}^{2+}{ }_{0.10} \mathrm{Mg}_{0.27}\right)_{2.04}\left(\mathrm{Si}_{3.68} \mathrm{Al}_{0.32}\right)_{4} \mathrm{O}_{10}(\mathrm{OH})_{2}, \mathrm{nH}_{2} \mathrm{O}
$$

The structural formula of the Giral glauconite indicates the dominance of iron into the octahedral site. The octahedral sheet contains 1.03 atoms per formula unit (a.p.f.u.) of $\mathrm{Fe}$ of which $\mathrm{Fe}^{3+}$ contributes to 0.93 a.p.f.u. and $\mathrm{Fe}^{2+}$ contributes to 0.10 a.p.f.u. The rest of the octahedral sites are occupied by $\mathrm{Al}^{3+}$ and $\mathrm{Mg}^{2+}$ contributing to 0.73 a.p.f.u. and 0.27 a.p.f.u., respectively. The sum of all octahedral cations varies between 2.00 and 2.18 (av. 2.04). The tetrahedral site is dominated by $\mathrm{Si}^{4+}$, which occupies 3.68 a.p.f.u. while $\mathrm{Al}^{3+}$ contributes to 0.32 a.p.f.u. for the tetrahedral site. The tetrahedral site is devoid of Fe. The interlayer cationic sites add up to 0.54 a.p.f.u. A total of $2 / 3 \mathrm{rd}$ of the interlayer cationic sites are occupied by $\mathrm{K}^{+}$while $\mathrm{Na}^{+}$contributes to the remaining $1 / 3 \mathrm{rd}$ interlayer sites with 0.36 and 0.18 a.p.f.u., respectively. 
Based on the $\mathrm{K}_{2} \mathrm{O}$ content, glauconite can be subdivided into nascent $\left(2-4 \mathrm{wt} \% \mathrm{~K}_{2} \mathrm{O}\right)$, slightly evolved (4-6 wt \% $\left.\mathrm{K}_{2} \mathrm{O}\right)$, evolved $\left(6-8 \mathrm{wt} \% \mathrm{~K}_{2} \mathrm{O}\right)$, and highly evolved $\left(>8 w \mathrm{t} \% \mathrm{~K}_{2} \mathrm{O}\right)$ glauconite [1]. Authigenic green pellets from the Giral Member, with low interlayer cation (av. 0.54 a.p.f.u.) and considerably high octahedral $\mathrm{Al}^{3+}$ content (av. 0.73 a.p.f.u.) belong to the slightly evolved variety. The X-ray diffraction parameters indicate a high degree of smectite interstratification, corroborating the slightly evolved nature of the glauconite. The low interlayer cation content corroborates that glauconite belongs to an immature variety. The Giral glauconite contains higher $\mathrm{Na}_{2} \mathrm{O}, \mathrm{Al}_{2} \mathrm{O}_{3}$, moderate $\mathrm{Fe}_{2} \mathrm{O}_{3}$ (total), and lower $\mathrm{K}_{2} \mathrm{O}$ contents than the ideal variety of glauconite reported by Odin and Matter $[1,54]$.

The Giral glauconite shows a moderate to high tetrahedral charge (T.C.), varying from -0.23 to -0.50 (av. -0.32 ). The octahedral charge (O.C.) shows low to moderate values, ranging from -0.02 to -0.33 (av. -0.25 ). The interlayer charge (I.C.) of the Giral glauconite shows low to high values, ranging from 0.37 to 0.62 (av. 0.54) (Supplementary Table S1). The interlayer charge exceeds 0.60 in matured glauconite, showing negligible smectite interstratification. The tetrahedral $\mathrm{R}^{3+}\left(\mathrm{Al}^{3+}\right)$ value of 0.32 a.p.f.u. and the octahedral $\mathrm{R}^{3+}$ $\left(\mathrm{Al}^{3+}+\mathrm{Fe}^{3+}\right)$ value of 1.76 a.p.f.u. are characteristic of glauconite mineral [55]. Geochemical analysis of Giral glauconite reflects a 'nascent' to 'slightly evolved' nature [1,4], which is corroborated by the low interlayer charge of the glauconite.

Due to the low interlayer cationic content, the Giral glauconite does not occupy the 'glauconite' zone demarcated by Meunier and El Albani [56] in the plot of dioctahedral Fe-rich clay minerals (Figure 8). The bivariant plot of $\mathrm{M}^{+} / 4 \mathrm{Si}$ vs. Fe/Sum of octahedra projection of dioctahedral Fe-rich clay minerals was later modified by Baldermann et al. [4] to incorporate the immature glauconitic smectite showing different maturation pathways of shallow and deep marine glauconitization (Figure 8). The Giral glauconite shows a cluster of data-points occupying the shallow marine glauconitization pathway suggesting evolution from an Al-rich substrate, and faster glauconitization reaction compared with that of the deep marine counterpart.

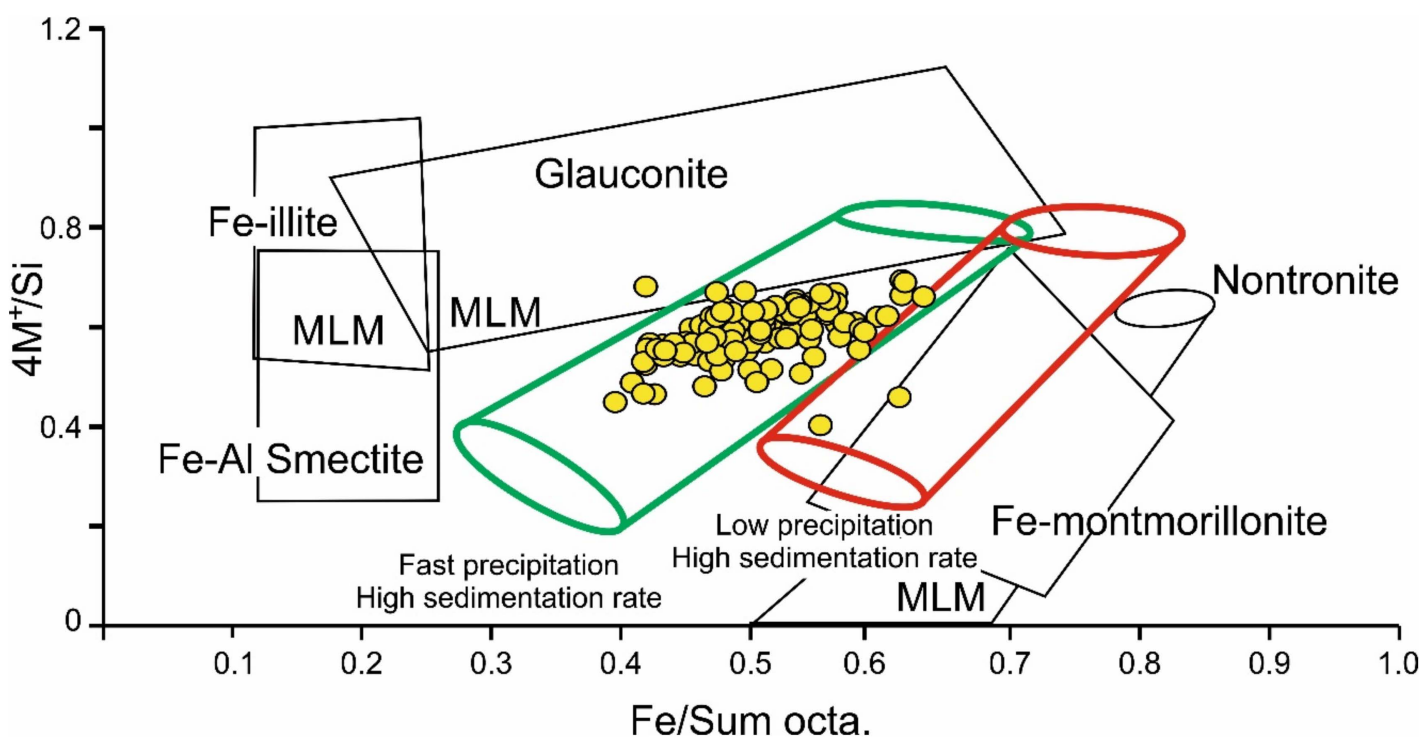

Figure $8.4 \mathrm{M}^{+} / \mathrm{Si}$ vs. Fe/sum of octa. (octa. = octahedral cations) cross-plot of glauconite pellets of Dharavi Formation. $\mathrm{M}^{+}$denotes the sum of interlayer atoms (in a.p.f.u.) and Fe denotes the sum of octahedrally coordinated Fe atoms (in a.p.f.u.). The zones of dioctahedral and trioctahedral minerals (glauconite, Fe-illite, Fe-Al smectite, and nontronite) are denoted after Meunier and El Albani [56] and the green and red zones mark the shallow and deep marine glauconitization pathways of Baldermann et al. [4]. (MLM = mixed layer mineral). 


\section{Discussion}

\subsection{Glauconite-Lignite Association of Paleogene Deposits in India}

The lignite and/or black shale deposits are fairly common within the Paleogene sediments [57-59]. Especially, the Paleocene-Eocene transition is marked by abundant lignite deposits along the continental margins, even in high-latitude sections [18,60]. Eocene glauconitic shale is common within the western passive margin basins in India, including Jaisalmer [19], Kutch [5,6,23], and Cambay basins [26,27]. The glauconite occurs within the limestone-shale alternation within the lignite-bearing deposits of the Giral Member. The floral and faunal assemblages, including terrestrial and marine palynomorphs and dinoflagellates, indicate an early Eocene age of the Giral Member [31,33]. The detailed biostratigraphic analysis of the Giral Member identifies the upper part of Apectodinium homomorphum cenozone corresponding to early Ypresian age for the glauconitic interval, immediately after the PETM at the Paleocene-Eocene transition. The Early Eocene (Ypresian) lignite-glauconite association also occurs within the Cambay Shale Formation of the adjacent Cambay basin [27,61] and the Naredi Formation of Kutch Basin [23,62-64]. Glauconite formed a few thousand years before the onset of PETM in the Jaisalmer Basin. Although the high abundance of dinocyst Kenleyia sp. characterizes the Early Eocene Climatic Optimum (EECO) within the lignite deposits of the adjacent Cambay Basin, the lignite-shale association of the Giral Member cannot be precisely linked to this event because of the paucity of benthic foraminifera [26,33]. The Paleogene lignite deposits mostly represent strandplain deposits in tropical, warm-humid climatic conditions while these time-equivalent glauconite deposits represent the subsequent marine flooding events.

\subsection{Unique Chemical Composition of Giral Glauconite and Origin of Glauconite}

The chemical composition of glauconite shows a wide range with variable $\mathrm{K}_{2} \mathrm{O}, \mathrm{Al}_{2} \mathrm{O}_{3}$, and $\mathrm{Fe}_{2} \mathrm{O}_{3}$ (total) content [1]. The $\mathrm{K}$-poor variety characterizes nascent glauconite while the K-rich variety characterizes highly matured glauconite $[1,3,4,7]$. The established range of composition of glauconite is defined as $2-10 w t \% \mathrm{~K}_{2} \mathrm{O}, 20-25 w \mathrm{t} \% \mathrm{Fe}_{2} \mathrm{O}_{3}$ (total), and 3-11 wt $\% \mathrm{Al}_{2} \mathrm{O}_{3}$ [65]. The chemistry of Giral glauconite is unique because of the high contents of $\mathrm{Na}_{2} \mathrm{O}$ (av. $1.33 \mathrm{wt} \%$ ), $\mathrm{Al}_{2} \mathrm{O}_{3}$, and $\mathrm{Fe}_{2} \mathrm{O}_{3}$ (total). The $\mathrm{Na}_{2} \mathrm{O}$ occupies the interlayer site of these glauconites and contributes to one third of the interlayer cation occupancy. While the $\mathrm{Al}_{2} \mathrm{O}_{3}$ content of glauconite shows consistently high values (av. $>10 \mathrm{wt} \%$ ) in the Precambrian deposits, a few Mesozoic and Cenozoic glauconites record high $\mathrm{Al}_{2} \mathrm{O}_{3}$ contents. The Giral glauconite, although containing low $\mathrm{K}_{2} \mathrm{O}$, have moderate $\mathrm{Fe}_{2} \mathrm{O}_{3}$ (total) and occupies the 'field of glauconite' of Odin and Matter [1] unlike most high-alumina glauconites which plot within the ferric illite field. Glauconite with high $\mathrm{Al}_{2} \mathrm{O}_{3}$ content $\left(>10 \mathrm{wt} \%\right.$ ) exhibits a low $(<10 \mathrm{wt} \%) \mathrm{Fe}_{2} \mathrm{O}_{3}$ (total) $[7,19]$. The elevated $\mathrm{Al}_{2} \mathrm{O}_{3}$ content of glauconite is often related to diagenetic alteration [66], sub-aerial weathering [67], and freshwater input. The inflow of freshwater in the marginal marine depositional site drastically reduces the $\mathrm{K}^{+}$activity, thus lowering the rate of glauconitization and giving rise to a K-poor glauconitic composition [68]. Short residence time of glauconite pellets may also contribute to the formation of K-poor glauconite, which cannot be distinguished from glauconite formations hindered by freshwater input [68]. Petrographic study rules out the possibility of diagenetic alteration or sub-aerial weathering of the glauconite. Absence of goethite and/or pyrite suggest similar sub-oxic condition prevailed throughout the glauconite formation [19]. The enhanced supply of organic matter from the continent might have facilitated the sub-oxic depositional condition where the formation of glauconite through incorporation of $\mathrm{K}$ and $\mathrm{Fe}$ by the substrate $[7,18,19,41,43]$. The high $\mathrm{Al}_{2} \mathrm{O}_{3}$ content of the Giral glauconite corresponding to the tetrahedral $\mathrm{Al}^{3+}$ suggests a high aluminous substrate for these glauconites [19]. The enriched $\mathrm{Na}_{2} \mathrm{O}$ content of Giral glauconite suggests a unique chemical pathway of maturation. Such abnormally high $\mathrm{Na}_{2} \mathrm{O}$ content in glauconite is rarely addressed [69-71]. The high Na content in glauconite may indicate the dissolution of volcaniclastic constituents, enriching the porewater [72]. Alternately, the chemical interaction with Na-rich groundwater can form Na-rich glauconite [73]. However, 
later alteration by groundwater would have formed a rim of an altered zone with Na-rich glauconite around the pellets. The Na content of Giral glauconite is evenly distributed within the grains. Further, salt deposits are absent within the Paleogene deposits of the Barmer basin. The predominance of lignite and carbonaceous shale within the Giral succession suggests a humid climatic condition. The late Paleocene-early Eocene time witnessed large-scale volcanism in the North Sea basin which is known as the North Atlantic Igneous Province, associated with several distinct glauconite deposits within the basin $[18,74]$. The euhedral quartz grains and the presence of feldspars with perthite textures (Figure 3D) suggest sediment inputs from volcanogenic sources adjacent to the Barmer Basin. Thus, the dissolution of volcaniclastics seems to be the most plausible source of high $\mathrm{Na}^{+}$within the Giral glauconites. The Na-rich porewater possibly led to the incorporation of $\mathrm{Na}^{+}$into the glauconite structure [72,75].

Although the $\mathrm{Al}_{2} \mathrm{O}_{3}$ content of the Giral glauconite is high, the $\mathrm{Fe}_{2} \mathrm{O}_{3} /\left(\mathrm{Fe}_{2} \mathrm{O}_{3}+\mathrm{Al}_{2} \mathrm{O}_{3}\right)$ ratio (av. 0.56) supports a 'ferruginous' character of these glauconites [66]. Similar glauconite, with considerably high $\mathrm{Al}_{2} \mathrm{O}_{3}$ and moderately high $\mathrm{Fe}_{2} \mathrm{O}_{3}$ (total), occurs within the Khuiala Formation from the adjacent Jaisalmer Basin forms by the reaction of kaolinite with iron oxide and minor smectite in an oxygen-depleted mid-shelf depositional environment [19]. The high tetrahedral $\mathrm{Al}^{3+}$ content corresponds to a highly aluminous substrate of the glauconite. The unique chemical composition and the abundance of glauconite within the Giral Member strongly suggest influence of warm climatic condition in its formation. The enhanced chemical weathering and run-off associated with warm-humid climatic condition resulted in supply of continental detritus, such as kaolinite and feldspar, within the shallow marine realm. Glauconite formation is also facilitated by the enhanced supply of nutrients such as $\mathrm{K}, \mathrm{Fe}$, and $\mathrm{Mg}$ in the shallow marine realm supplied as dissolved or particulate matter $[18,19,76]$. However, the $\mathrm{K}^{+}$content of Giral glauconite is lower than the Khuiala glauconite. The low $\mathrm{K}_{2} \mathrm{O}$ content, therefore, reflects a combination of freshwater input in a marginal marine depositional environment and a relatively high rate of sedimentation discouraging the maturation of glauconite.

\subsection{Relationship of Glauconite Formation and Iron Sequestration}

Recent review on biostratigraphically constrained sections reveal the high abundance of glauconite associated with early, middle and late Paleogene time, closely corresponding to warm climatic intervals of Paleogene [18]. Continental erosion, enhanced during warm climatic conditions, is responsible for the abundant supply of elements such as $\mathrm{K}, \mathrm{Fe}, \mathrm{Al}$, $\mathrm{Si}, \mathrm{P}$, and $\mathrm{Mg}$, which influences the ocean geochemistry [76]. Increased supply of organic matter and its subsequent decomposition results in oxygen-depletion in the shallow marine environment, favoring the reduction of Fe-particulate matter to mobile Fe(II) state and its fixation into glauconite structure $[6,7,18,19]$. Since iron is a major limiting factor in ocean productivity, its supply and sequestration play an important role in the biogeochemical evolution of the ocean. While anoxic-sulphidic depositional environments are suitable for fast sequestration of iron as Fe-sulphides, recent investigations indicate a significant contribution of Fe-silicates authigenesis (especially glauconite) in iron sequestration in geological record [17]. The restricted marine Giral Member represents a marine incursion in response to the eustatic sea-level rise at the earliest part of the Eocene [33,77]. Glauconitic deposits in adjacent Cambay (Cambay Shale Formation), Kutch (Naredi Formation), and Jaisalmer (Khuiala Formation) suggest favorable ocean water geochemistry [19]. The process of glauconitization from an aluminous clay substrate to glauconite formation through simultaneous intake of $\mathrm{K}$ and Fe may have contributed substantially to sequestrating iron within the sedimentary column. Glauconite authigenesis in shallow marine conditions can be effective in sequestrating $\sim 30 \%$ of the particulate and dissolved iron supplied by the riverine flux [78]. Recent investigation on deep marine sediments from the ODP site 959 suggests a Fe-sequestration rate of $\sim 80 \mu \mathrm{mol} \mathrm{cm}{ }^{-2} \cdot \mathrm{kyr}^{-1}$, which is on average six times higher than pyritization [17]. Considering the widespread glauconitization along the 
western Indian passive margin, we can conceptualize significant Fe-sequestration during the Paleocene-Eocene transition.

\section{Conclusions}

The major conclusions of this study are:

a. The lignite-bearing, Early Eocene succession of the Giral Member of the Barmer Basin hosts a marine flooding event represented by abundant glauconite formation.

b. Mineralogical and micro-textural data indicate the 'nascent' to 'slightly evolved' variety of glauconite, suggesting a combination of dilution by freshwater inflow and relatively high sedimentation rate in the marginal marine environment.

c. The Giral glauconite is compositionally unique because of its high $\mathrm{Al}_{2} \mathrm{O}_{3}$ and $\mathrm{Na}_{2} \mathrm{O}$ and moderately high $\mathrm{Fe}_{2} \mathrm{O}_{3}$ (total) content. The dissolution of volcaniclastic possibly induced the formation of Na-rich glauconite, by enriching the porewater with $\mathrm{Na}$.

d. The warm climate at the earliest part of the Eocene facilitated the glauconitization through the enhanced supply of elements such as Fe, Al, Si, K, and P due to increased weathering and run-off on the continent, turning the shallow marine environment sub-oxic through rapid oxidation of organic matter.

e. Glauconite authigenesis in the Giral Member could be a part of the potential sink for iron in the shallow, marginal marine depositional environment during the Early Eocene.

Supplementary Materials: The following are available online at https:/ /www.mdpi.com/article/ 10.3390/min12010056/s1. Table S1: Cation distribution in glauconite structure.

Author Contributions: Data curation, T.R.C., S.K. and S.S.M.; formal analysis, T.R.C., S.K. and S.S.M.; investigation, S.B. and T.R.C.; methodology, T.R.C. and S.S.M.; supervision, S.B.; validation, S.K.; writing-original draft, T.R.C.; writing-review \& editing, S.B. All authors have read and agreed to the published version of the manuscript.

Funding: Council of Scientific and Industrial Research (CSIR) provided the fund to T.R.C. for research work. Department of Science and Technology (DST), India provided grant (INT/RUS/RFBR/390) to S.B. for analysis related to this research.

Data Availability Statement: Not Applicable.

Conflicts of Interest: The authors declare no conflict of interest.

\section{References}

1. Odin, G.S.; Matter., A. De glauconiarum origine. Sedimentology 1981, 28, 611-641. [CrossRef]

2. Tribovillard, N.; Bout-Roumazeilles, V.; Delattre, M.; Ventalon, S.; Abraham, R.; Nzié, O. Syndepositional glauconite as a paleoenvironmental proxy-the lower Cenomanian Chalk of Cap Blanc Nez (N-France). Chem. Geol. 2021, 584, 120508. [CrossRef]

3. Baldermann, A.; Warr, L.N.; Grathoff, G.H.; Dietzel, M. The rate and mechanism of deep-sea glauconite formation at the Ivory Coast-Ghana Marginal Ridge. Clays Clay Miner. 2013, 61, 258-276. [CrossRef]

4. Baldermann, A.; Dietzel, M.; Mavromatis, V.; Mittermayr, F.; Warr, L.N.; Wemmer, K. The role of Fe on the formation and diagenesis of interstratified glauconite-smectite and illite-smectite: A case study of Upper Cretaceous shallow-water carbonates. Chem. Geol. 2017, 453, 21-34. [CrossRef]

5. Banerjee, S.; Chattoraj, S.L.; Saraswati, P.K.; Dasgupta, S.; Sarkar, U. Mineralogy and geochemistry of lagoonal glauconites and their implications on origin and maturation: Oligocene Maniyara Fort Formation, western Kutch, India. Geol. J. 2012, 47, 357-371. [CrossRef]

6. Banerjee, S.; Chattoraj, S.L.; Saraswati, P.K.; Dasgupta, S.; Sarkar, U. Substrate control on formation and maturation of glauconites in the Middle Eocene Harudi Formation, western Kutch India. Mar. Petrol. Geol. 2012, 30, 144-160. [CrossRef]

7. Banerjee, S.; Bansal, U.; Thorat, A. A review on palaeogeographic implications and temporal variation in glaucony composition. J. Palaeogeogr. 2016, 5, 43-71. [CrossRef]

8. Huggett, J.; Adetunji, J.; Longstaffe, F.; Wray, D. Mineralogical and geochemical characterisation of warm-water, shallow-marine glaucony from the Tertiary of the London Basin. Clay Miner. 2017, 52, 25-50. [CrossRef]

9. Giresse, P.; Bayon, G.; Tallobre, C.; Loncke, L. Neodymium isotopes in glauconite for palaeoceanographic reconstructions at continental margins: A preliminary investigation from Demerara Rise. Front. Earth Sci. 2021, 9, 1-14. [CrossRef] 
10. López-Quirós, A.; Sánchez-Navas, A.; Nieto, F.; Escutia, C. New insights into the nature of glauconite. Am. Miner. 2020, 105, 674-686. [CrossRef]

11. López-Quirós, A.; Escutia, C.; Sanchez-Navas, A.; Nieto, F.; García-Casco, A.; Martín-Algarra, A.; Evangelinos, D.; Salabarnada, A. Glaucony authigenesis, maturity and alteration in the Weddell Sea: An indicator of paleoenvironmental conditions before the onset of Antarctic glaciation. Sci. Rep. 2019, 9, 13580. [CrossRef] [PubMed]

12. Sánchez-Navas, A.; Algarra, A.M.; Eder, V.; Reddy, B.J.; Nieto, F.; Zanin, Y.N. Color, mineralogy and composition of Upper Jurassic West Siberian glauconite: Useful indicators of paleoenvironment. Can. Miner. 2008, 46, 1545-1564. [CrossRef]

13. Jimenez-Millan, J.; Molina, J.M.; Nieto, F.; Nieto, L.; Ruiz-Ortiz, P.A. Glauconite and phosphate peloids in Mesozoic carbonate sediments (eastern Subbetic Zone, Betic Cordilleras, SE Spain). Clay Miner. 1998, 33, 547-559. [CrossRef]

14. Wigley, R.; Compton, J.S. Oligocene to Holocene glauconite-phosphorite grains from the Head of the Cape Canyon on the western margin of South Africa. Deep. Sea Res. Part II Top. Stud. Oceanogr. 2007, 54, 1375-1395. [CrossRef]

15. Stille, P.; Clauer, N. The process of glauconitization: Chemical and isotopic evidence. Contrib. Miner. Petrol. 1994, 117, 253-262. [CrossRef]

16. Udgata, D.B.P. Glauconite as an Indicator of Sequence Stratigraphic Packages in a Lower Paleocene Passive-Margin Shelf Succession, Central Alabama. Master's Thesis, Auburn University, Auburn, AL, USA, 2007; 109p.

17. Baldermann, A.; Warr, L.N.; Letofsky-Papst, I.; Mavromatis, V. Substantial iron sequestration during green-clay authigenesis in modern deep-sea sediments. Nat. GeoSci. 2015, 8, 885-889. [CrossRef]

18. Banerjee, S.; Roy Choudhury, T.; Saraswati, P.K.; Khanolkar, S. The formation of authigenic deposits during Paleogene warm climatic intervals: A review. J. Palaeogeogr. 2020, 9, 27. [CrossRef]

19. Roy Choudhury, T.; Banerjee, S.; Khanolkar, S.; Saraswati, P.K. Glauconite authigenesis during the onset of the Paleocene-Eocene Thermal Maximum: A case study from the Khuiala Formation in Jaisalmer Basin, India. Palaeogeogr. Palaeocl. 2021, 571, 110388. [CrossRef]

20. John, C.M.; Bohaty, S.M.; Zachos, J.C.; Sluijs, A.; Gibbs, S.; Brinkhuis, H.; Bralower, T.J. North American continental margin records of the Paleocene-Eocene thermal maximum: Implications for global carbon and hydrological cycling. Paleoceanography 2008, 23, PA2217. [CrossRef]

21. Sluijs, A.; Van Roij, L.; Harrington, G.J.; Schouten, S.; Sessa, J.A.; LeVay, L.J.; Reichart, G.J.; Slomp, C.P. Warming, euxinia and sea level rise during the Paleocene-Eocene Thermal Maximum on the Gulf Coastal Plain: Implications for ocean oxygenation and nutrient cycling. Clim. Past 2014, 10, 1421-1439. [CrossRef]

22. Singh, N.P. Cenozoic lithostratigraphy of the Jaisalmer basin, Rajasthan. J. Palaeontol. Soc. India 2007, 52, 129-154.

23. Chattoraj, S.; Banerjee, S.; Saraswati, P.K. Glauconites from the late Palaeocene Early Eocene Naredi Formation, Western Kutch and their genetic implications. J. Geol. Soc. India 2009, 73, 567-574. [CrossRef]

24. Chattoraj, S.L.; Banerjee, S.; Saraswati, P.K.; Bansal, U. Origin, depositional setting and stratigraphic implications of Palaeogene glauconite of Kutch. Spec. Publ. Geol. Soc. India 2016, 6, 75-88.

25. Chakrabarty, S.; Gorai, D.; Shukla, M.; Uppal, S. High Resolution Sequence Stratigraphy and its Implication in Mixed Siliciclastic Carbonate Sequence: A Case Study from Early to Middle Eocene Sylhet Formation, Assam and Assam-Arakan Basin, India. In Proceedings of the AAPG International Conference and Exhibition, Cape Town, South Africa, 4-7 November 2018.

26. Clementz, M.; Bajpai, S.; Ravikant, V.; Thewissen, J.G.M.; Saravanan, N.; Singh, I.B.; Prasad, V. Early Eocene warming events and the timing of terrestrial faunal exchange between India and Asia. Geology 2011, 39, 15-18. [CrossRef]

27. Samanta, A.; Bera, M.K.; Ghosh, R.; Bera, S.; Filley, T.; Pande, K.; Rathore, S.S.; Rai, J.; Sarkar, A. Do the large carbon isotopic excursions in terrestrial organic matter across Paleocene-Eocene boundary in India indicate intensification of tropical precipitation? Palaeogeogr. Palaeocl. 2013, 387, 91-103. [CrossRef]

28. Khanolkar, S.; Roy Choudhury, T.; Saraswati, P.K.; Banerjee, S. Late Paleocene-early Eocene foraminiferal assemblage and carbon isotope excursion indicating hyperthermal events in paleotropical succession of northwestern India. J. Foraminifer. Res. 2021, 51, 4-13. [CrossRef]

29. Tripathi, S.K.M.; Kumar, M.; Srivastava, D. Palynology of lower palaeogene (Thanetian-Ypresian) coastal deposits from the Barmer Basin (Akli Formation, Western Rajasthan, India): Palaeoenvironmental and palaeoclimatic implications. Geol. Acta 2009, 7, 147-160.

30. Compton, P.M. The geology of the Barmer Basin, Rajasthan, India, and the origins of its major oil reservoir, the Fatehgarh Formation. Petrol. Geosci. 2009, 15, 117-130. [CrossRef]

31. Dolson, J.; Burley, S.D.; Sunder, V.R.; Kothari, V.; Naidu, B.; Whiteley, N.P.; Farrimond, P.; Taylor, A.; Direen, N.; Ananthakrishnan, B. The discovery of the Barmer Basin, Rajasthan, India, and its petroleum geology. AAPG Bull. 2015, 99, 433-465. [CrossRef]

32. Sisodia, M.S.; Singh, U.K. Depositional environment and hydrocarbon prospects of the Barmer Basin, Rajasthan, India. Nafta 2000, 51, 309-326

33. Khanolkar, S.; Sharma, J. Record of early to middle Eocene paleoenvironmental changes from lignite mines, western India. $J$. MicroPalaeontol. 2019, 38, 1-24. [CrossRef]

34. Rana, R.S.; Kumar, K.; Singh, H.; Rose, K.D. Lower vertebrates from the late Palaeocene-earliest Eocene Akli formation, giral lignite mine, Barmer District, western India. Curr. Sci. India 2005, 89, 1606-1613.

35. Tripathi, S.K.M.; Srivastava, D. Palynological investigations, facies analysis and palaeoenvironmental interpretations from Late Palaeocene to Early Eocene lignites and associated sediments of Barmer, western India. Palaeobotanist 2010, 59, 1-32. 
36. Singh, H. Palynofloral investigation of the Akli Formation (Palaeocene) of Giral lignite mine, Barmer district, Rajasthan. Geophytology 2015, 45, 209-214.

37. Prasad, V.; Uddandam, P.R.; Agrawal, S.; Bajpai, S.; Mishra, I.S.A.K.; Sharma, A.; Verma, M.K.P. Biostratigraphy, palaeoenvironment and sea level changes during pre-collisional (Palaeocene) phase of the Indian plate: Palynological evidence from Akli Formation in Giral Lignite Mine, Barmer Basin, Rajasthan, Western India. Epis. J. Int. Geosci. 2020, 43, 476-488. [CrossRef]

38. Tripathi, S.K.M.; Singh, U.K.; Sisodia, M.S. Palynological investigation and environmental interpretation on Akli Formation (late Palaeocene) from Barmer Basin, western Rajasthan, India. Palaeobotanist 2003, 52, 87-95.

39. Garg, R.; Ateequzzaman, K.; Prasad, V.; Tripathi, S.K.M.; Singh, I.B.; Jauhri, A.K.; Bajpai, S. Age-diagnostic dinoflagellate cysts from the lignite-bearing sediments of the Vastan lignite mine, Surat District, Gujarat, western India. J. Palaeontol. Soc. India 2008, 53, 99-105.

40. Bansal, U.; Banerjee, S.; Pande, K.; Arora, A.; Meena, S.S. The distinctive compositional evolution of glauconite in the Cretaceous Ukra Hill Member (Kutch basin, India) and its implications. Mar. Petrol. Geol. 2017, 82, 97-117. [CrossRef]

41. Tounekti, A.; Boukhalfa, K.; Roy Choudhury, T.; Soussi, M.; Banerjee, S. Global and local factors behind the authigenesis of Fe-silicates (Glauconite/Chamosite) in Miocene strata of Northern Tunisia. J. Afr. Earth Sci. 2021, 184, 104342. [CrossRef]

42. Fritz, W.J.; Howells, M.F. A shallow marine volcaniclastic facies model: An example from sedimentary rocks bounding the subaqueously welded Ordovician Garth Tuff, North Wales, UK. Sediment. Geol. 1991, 74, 217-240. [CrossRef]

43. Banerjee, S.; Farouk, S.; Nagm, E.; Roy Choudhury, T.; Meena, S.S. High Mg-glauconite in Campanian Duwi Formation of Abu Tartur Plateau, Egypt and its implications. J. Afr. Earth Sci. 2019, 156, 12-25. [CrossRef]

44. Thompson, G.R.; Hower, J. The mineralogy of glauconite. Clays Clay Miner. 1975, 23, 289-300. [CrossRef]

45. Amorosi, A.; Sammartino, I.; Tateo, F. Evolution patterns of glaucony maturity: A mineralogical and geochemical approach. Deep. Sea Res. Part II Top. Stud. Oceanogr. 2007, 54, 1364-1374. [CrossRef]

46. Odom, I.E. Glauconite and celadonite minerals. Rev. Miner. 1984, 13, 545-572.

47. Moore, D.M.; Reynolds, R.C.J. X-ray Diffraction and the Identification and Analysis of Clay Minerals, 1st ed.; Oxford University Press: Oxford, UK, 1989.

48. Banerjee, S.; Mondal, S.; Chakraborty, P.P.; Meena, S.S. Distinctive compositional characteristics and evolutionary trend of Precambrian glaucony: Example from Bhalukona Formation, Chhattisgarh basin, India. Precambrian Res. 2015, 271, 33-48. [CrossRef]

49. Heller-Kallai, L.; Rozenson, I. The use of Mössbauer spectroscopy of iron in clay mineralogy. Phys. Chem. Miner. 1981, 7, 223-238. [CrossRef]

50. Cardile, C.M.; Brown, I.W.M. An ${ }^{57}$ Fe Mössbauer spectroscopic and X-ray diffraction study of New Zealand glauconites. Clay Miner. 1988, 23, 13-25. [CrossRef]

51. Drits, V.A.; Dainyak, L.G.; Muller, F.; Besson, G.; Manceau, A. Isomorphous cation distribution in celadonites, glauconites and Fe-illites determined by infrared, Mössbauer and EXAFS spectroscopies. Clay Miner. 1997, 32, 153-179. [CrossRef]

52. Amouric, M.; Parron, C. Structure and growth mechanism of glauconite as seen by high-resolution transmission electron microscopy. Clays Clay Miner. 1985, 33, 473-482. [CrossRef]

53. Baldermann, A.; Grathoff, G.H.; Nickel, C. Micromilieu-controlled glauconitization in fecal pellets at Oker (Central Germany). Clay Miner. 2012, 47, 513-538. [CrossRef]

54. Amorosi, A. Detecting compositional, spatial, and temporal attributes of glaucony: A tool for provenance research. Sediment. Geol. 1997, 109, 135-153. [CrossRef]

55. Bailey, S.W. Summary of recommendations of AIPEA nomenclature committee. Clay Miner. 1980, 15, 85-93. [CrossRef]

56. Meunier, A.; El Albani, A. The glauconite-Fe-illite-Fe-smectite problem: A critical review. Terra Nova 2007, 19, 95-104. [CrossRef]

57. Bolle, M.P.; Pardo, A.; Hinrichs, K.U.; Adatte, T.; Von Salis, K.; Burns, S.; Keller, G.; Muzylev, N. The Paleocene-Eocene transition in the marginal northeastern Tethys (Kazakhstan and Uzbekistan). Int. J. Earth Sci. 2000, 89, 390-414. [CrossRef]

58. Gavrilov, Y.O.; Shcherbinina, E.A.; Golovanova, O.V.; Pokrovskii, B.G. The late Cenomanian paleoecological event (OAE 2) in the eastern Caucasus basin of Northern Peri-Tethys. Lithol. Miner. Resour. 2013, 48, 457-488. [CrossRef]

59. Schulte, P.; Schwark, L.; Stassen, P.; Kouwenhoven, T.J.; Bornemann, A.; Speijer, R.P. Black shale formation during the Latest Danian Event and the Paleocene-Eocene Thermal Maximum in central Egypt: Two of a kind? Palaeogeogr. Palaeocl. 2013, 371, 9-25. [CrossRef]

60. Dypvik, H.; Riber, L.; Burca, F.; Rüther, D.; Jargvoll, D.; Nagy, J.; Jochmann, M. The Paleocene-Eocene thermal maximum (PETM) in Svalbard-Clay mineral and geochemical signals. Palaeogeogr. Palaeocl. 2011, 302, 156-169. [CrossRef]

61. Samanta, A.; Sarkar, A.; Bera, M.K.; Rai, J.; Rathore, S.S. Late Paleocene-early Eocene carbon isotope stratigraphy from a near-terrestrial tropical section and antiquity of Indian mammals. J. Earth Syst. Sci. 2013, 122, 163-171. [CrossRef]

62. Garg, R.; Prasad, V.; Thakur, B.; Singh, I.B.; Khowaja-Ateequzzaman. Dinoflagellate cysts from the Naredi Formation, southwestern Kutch, India: Implication on age and palaeoenvironment. J. Palaeontol. Soc. India 2011, 56, 201-218.

63. Saraswati, P.K.; Khanolkar, S.; Raju, D.S.N.; Dutta, S.; Banerjee, S.; Wang, Y.; Liu, M. Foraminiferal biostratigraphy of lignite mines of Kutch India: Age of lignite fossil vertebrates. J. Palaeogeogr. 2014, 3, 90-98.

64. Banerjee, S.; Khanolkar, S.; Saraswati, P.K. Facies and depositional settings of the Middle Eocene-Oligocene carbonates in Kutch. Geodin Acta 2018, 30, 119-136. [CrossRef] 
65. Odin, G.S.; Fullagar, P.D. Geological significance of the glaucony facies. In Developments in Sedimentology; Odin, G.S., Ed.; Elsevier: Paris, France, 1988; Volume 45.

66. Jach, R.; Starzec, K. Glaucony from the condensed Lower-Middle Jurassic deposits of the Križna Unit, Western Tatra Mountains, Poland. Ann. Soc. Geol. Pol. 2003, 73, 183-192.

67. Skiba, M.; Maj-Szeliga, K.; Szymański, W.; Błachowski, A. Weathering of glauconite in soils of temperate climate as exemplified by a Luvisol profile from Góra Puławska, Poland. Geoderma 2014, 235, 212-226. [CrossRef]

68. El Albani, A.; Meunier, A.; Fürsich, F. Unusual occurrence of glauconite in a shallow lagoonal environment (Lower Cretaceous, northern Aquitaine Basin, SW France). Terra Nova 2005, 17, 537-544. [CrossRef]

69. Foster, M.D. Studies of Celadonite and Glauconite; United States Government Printing Office: Washington, DC, USA, 1969; pp. F1-F17.

70. Morton, A.C.; Merriman, R.J.; Mitchell, J.G. Genesis and significance of glauconitic sediments of the Southwest Rockall Plateau. Geology 1984, 81, 645-652.

71. McConchie, D.M.; Lewis, D.W. Authigenic, perigenic, and allogenic glauconites from the Castle Hill Basin, North Canterbury, New Zealand. N. Z. J. Geol. Geop. 1978, 21, 199-214. [CrossRef]

72. Monteiro, F.M.; Fonseca, M.M.; Madeira, M.A.; Herbillon, A.J. Driving factors determining the occurrence of sodic soils in dry subhumid Mediterranean areas. J. Plant Nutr. Soil Sci. 2012, 175, 94-100. [CrossRef]

73. Chapelle, F.H.; Knobel, L.L. Aqueous geochemistry and the exchangeable cation composition of glauconite in the Aquia aquifer, Maryland. Groundwater 1983, 21, 343-352. [CrossRef]

74. Jones, M.T.; Percival, L.M.; Stokke, E.W.; Frieling, J.; Mather, T.A.; Riber, L.; Schubert, B.A.; Schultz, B.; Tegner, C.; Planke, S.; et al. Mercury anomalies across the Palaeocene-Eocene thermal maximum. Clim. Past 2019, 15, 217-236. [CrossRef]

75. Barton, J.M., Jr.; Cairncross, B.; McLachlan, I. Rb-Sr isotopic and elemental studies of the origin of glauconite in the Permian northern Karoo Coal Fields, South Africa: Evidence for a thermal Mid-Jurassic influence. S. Afr. J. Geol. 2004, 107, 499-504. [CrossRef]

76. Peters, S.E.; Gaines, R.R. Formation of the 'Great Unconformity' as a trigger for the Cambrian explosion. Nature 2012, 484, 363-366. [CrossRef] [PubMed]

77. Sahni, A.; Rana, R.S.; Loyal, R.S.; Saraswati, P.K.; Mathur, S.K.; Rose, K.D.; Tripathi, S.K.M.; Garg, R. Western margin Palaeocenelower Eocene lignites: Biostratigraphic and palaeoecological constraints. In Proceedings of the 2nd APG Conference cum Exhibition, Khajuraho, India, 24-26 September 2004; pp. 1-22.

78. Raiswell, R. Towards a global highly reactive iron cycle. J. Geochem. Explor. 2006, 88, 436-439. [CrossRef] 